DOI 10.4171/JEMS/280

Anne-Laure Dalibard

\title{
Stability of periodic stationary solutions of scalar conservation laws with space-periodic flux
}

Received April 6, 2009 and in revised form June 9, 2010

\begin{abstract}
This article investigates the long-time behaviour of parabolic scalar conservation laws of the type $\partial_{t} u+\operatorname{div}_{y} A(y, u)-\Delta_{y} u=0$, where $y \in \mathbb{R}^{N}$ and the flux $A$ is periodic in $y$. More specifically, we consider the case when the initial data is an $L^{1}$ disturbance of a stationary periodic solution. We show, under polynomial growth assumptions on the flux, that the difference between $u$ and the stationary solution behaves in $L^{1}$ norm like a self-similar profile for large times. The proof uses a time and space change of variables which is well-suited for the analysis of the long time behaviour of parabolic equations. Then, convergence in rescaled variables follows from arguments from dynamical systems theory. One crucial point is to obtain compactness in $L^{1}$ on the family of rescaled solutions; this is achieved by deriving uniform bounds in weighted $L^{2}$ spaces.
\end{abstract}

Keywords. Long time asymptotics, parabolic scalar conservation law, asymptotic expansion, moment estimates, homogenization

\section{Introduction}

The goal of this article is to study the long time limit of solutions of the equation

$$
\partial_{t} u+\operatorname{div}_{y} A(y, u)-\Delta_{y} u=0, \quad t>0, y \in \mathbb{R}^{N},
$$

where the flux $A: \mathbb{R}^{N} \times \mathbb{R} \rightarrow \mathbb{R}^{N}$ is assumed to be $\mathbb{T}^{N}$-periodic with respect to its first variable. Here and in the rest of the article, $\mathbb{T}^{N}$ denotes the $N$-dimensional torus, i.e. $\mathbb{T}^{N}=(\mathbb{R} / \mathbb{Z})^{N}$.

Classical results on scalar conservation laws (see for instance [21, 15]) ensure that the semigroup associated with equation (1.1) is well-defined in $L^{1}\left(\mathbb{R}^{N}\right)+L^{\infty}\left(\mathbb{R}^{N}\right)$. The case when the initial data belongs to $U(y)+L^{1}(\mathbb{R})$ (when $N=1$ ), where $U$ is a viscous shock profile of equation (1.1) has already been dealt with in a previous article (see [8]). In the present paper, we restrict our study to the case when the inital data belongs to $v(y)+L^{1}\left(\mathbb{R}^{N}\right)$, where $v$ is a given periodic stationary solution of (1.1). The choice of such initial data stems from the fact that the semigroup associated with (1.1) is nonexpansive in the $L^{1}$ norm; hence it is natural to investigate stability in $L^{1}\left(\mathbb{R}^{N}\right)$.

A.-L. Dalibard: CNRS, UMR 8553-DMA, École Normale Supérieure,

45 rue d’Ulm, 75005 Paris, France; e-mail: Anne-Laure.Dalibard@ens.fr

Mathematics Subject Classification (2010): 35B35, 35B40, 35B27 
When the flux $A$ is linear, say

$$
A(y, u)=\alpha(y) u,
$$

this study coincides (at least for some particular functions $\alpha$ ) with the one led by Adrien Blanchet, Jean Dolbeault and Michał Kowalczyk in [5] on the large time behaviour of Brownian ratchets, as we will explain in Remark 1.4. It is proved in [5] that if the flux $A$ is linear and if

$$
\limsup _{t \rightarrow \infty} \frac{1}{(1+2 t)^{2}} \int_{\mathbb{R}^{N}}|u(t, y)-v(y)|(y-c t)^{4} d y<\infty
$$

for some velocity $c \in \mathbb{R}^{N}$ which will be defined later on (see (1.7)), then there exists a constant $C$ and a number $\kappa \in(0,1 / 2)$ such that for all $t>0$,

$$
\int_{\mathbb{R}^{N}}\left|u(t, y)-v(y)-\frac{M f_{0}(y)}{(1+2 t)^{N / 2}} F\left(\frac{y-c t}{\sqrt{1+2 t}}\right)\right| d y \leq C t^{-\kappa},
$$

where $f_{0}$ is the solution of an elliptic equation in $\mathbb{T}^{N}$ (see (1.6)), $F$ is a Gaussian profile, and $M$ is the mass of the initial disturbance, i.e.

$$
M=\int_{\mathbb{R}^{N}}\left(u_{\mid t=0}-v\right)
$$

Inequality (1.3) is a technical assumption which is expected-but has yet to be proved-to hold for a large class of initial data.

Unfortunately, as we explained in [8], the above result does not imply that the same convergence holds in the nonlinear case. Moreover, the proof of [5], which is based on entropy dissipation methods together with log-Sobolev Poincaré inequalities, can hardly be transposed as such to a nonlinear setting, although attempts in this direction have been made: for instance, in [10] M. Di Francesco and P. Markowich prove convergence towards diffusive waves for the Burgers equation using entropy dissipation methods. However, their strategy relies crucially on the Hopf-Cole formula, and therefore could not be transposed to the present general setting. Hence we have chosen here a slightly different approach, which enables us to extend some of the results of [5] to a nonlinear context. Additionally, we recover a weaker version of the convergence (1.3) (with no convergence rate), but without the need for assumption (1.2). In fact, we prove that (1.2) holds for a class of initial data which is dense in $v+L^{1}\left(\mathbb{R}^{N}\right)$.

The present work is also embedded in the broader study of the long time behaviour of conservation laws. We refer the interested reader to the review paper by D. Serre [22] (and the references therein) for a thorough description of the homogeneous case, in which the author investigates the stability of stationary solutions of scalar conservation laws in various models (parabolic and hyperbolic settings, relaxation models, ...)

Before stating the main results of this paper, let us now recall a few properties of equation (1.1). First, according to a result of [7], periodic stationary solutions of (1.1) exist, provided the flux $A$ satisfies some growth assumptions. In fact, several different growth regimes were studied in [7]; we only recall one of them here, which is the most 
relevant with regards to our purposes. In the rest of the article, we assume that $A$ belongs to $W_{\text {loc }}^{1, \infty}\left(\mathbb{T}^{N} \times \mathbb{R}\right)^{N}$, and that

$$
\exists p_{0} \in \mathbb{R}, \forall y \in \mathbb{T}^{N}, \quad \operatorname{div}_{y} A\left(y, p_{0}\right)=0 .
$$

We also assume that there exists $n \in(0,(N+2) / N)$ such that

$$
\begin{aligned}
& \forall P>0, \exists C_{P}>0, \quad \forall(p, q) \in \mathbb{R}^{2},|p| \leq P, \\
& \qquad \begin{array}{l}
\left|\partial_{p} A(y, p+q)-\partial_{p} A(y, p)\right| \leq C_{P}\left(|q|+|q|^{n}\right), \\
\left|\operatorname{div}_{y} A(y, p+q)-\operatorname{div}_{y} A(y, p)\right| \leq C_{P}\left(|q|+|q|^{n}\right) .
\end{array}
\end{aligned}
$$

These assumptions were introduced in $[8,7]$. They ensure that for any $r \in \mathbb{R}$, there exists a unique periodic stationary solution of (1.1) with mean value $r$; we refer to [7] for a discussion of the optimality of conditions (1.4), (1.5). Moreover, if $u$ is a solution of (1.1) with initial data $u_{\mid t=0} \in v+L^{1} \cap L^{\infty}\left(\mathbb{R}^{N}\right)$, where $v \in W^{1, \infty}\left(\mathbb{R}^{N}\right)$ is any stationary solution of $(1.1)$, then $u \in L^{\infty}\left([0, \infty) \times \mathbb{R}^{N}\right)$. This result will be used several times in the article, and its proof is recalled in the Appendix.

We now introduce the profiles which characterize the asymptotic behaviour of the function $u$ :

- First, the function $f_{0}$ occurring in (1.3) is the unique solution in $H^{1}\left(\mathbb{T}^{N}\right)$ of the equation

$$
-\Delta_{y} f_{0}+\operatorname{div}_{y}\left(\alpha_{1} f_{0}\right)=0, \quad\left\langle f_{0}\right\rangle=1,
$$

where

$$
\alpha_{1}(y):=\left(\partial_{p} A\right)(y, v(y)) \in L^{\infty}\left(\mathbb{T}^{N}\right)^{N} .
$$

Above and in the rest of the article, the notation $\langle\cdot\rangle$ stands for the average on the torus $\mathbb{T}^{N}$, that is,

$$
\langle f\rangle:=\int_{\mathbb{T}^{N}} f .
$$

- The drift velocity $c$ is then defined by

$$
c=\left\langle\alpha_{1} f_{0}\right\rangle .
$$

- The last function which will appear in the asymptotic profile of $u$ is the equivalent, in the nonlinear case, of the Gaussian profile $F$ occurring in (1.3); it is the unique solution, in a suitable function space, of an elliptic equation of the form

$-\sum_{1 \leq i, j \leq N} \eta_{i, j} \partial_{i} \partial_{j} F_{M}-\operatorname{div}_{x}\left(x F_{M}\right)+a \cdot \nabla_{x} F_{M}^{2}=0$ in $\mathbb{R}^{N}$, with $\int_{\mathbb{R}^{N}} F_{M}=M \in \mathbb{R}$,

where the coefficients $\eta_{i, j}$ and $a$ are constant, and the matrix $\left(\eta_{i, j}\right)_{1 \leq i, j \leq N}$ is coercive. Unfortunately, giving the precise definition of $\eta_{i, j}$ and $a$ would take us too far at this stage. Let us merely mention that $a=0$ if $N \geq 2$. We also recall that thanks to a result of J. Aguirre, M. Escobedo, and E. Zuazua (see [1]), the above equation has a unique solution for all $M \in \mathbb{R}$, and we refer to the next section for more details. In particular, $\eta_{i, j}, a$ are defined in (2.14), (2.16) respectively. 
The main result of this paper is the following:

Theorem 1.1. Let $A \in W_{\mathrm{loc}}^{5, \infty}\left(\mathbb{T}^{N} \times \mathbb{R}\right)^{N}$, and assume that $A$ satisfies (1.4), (1.5). Let $v$ be a periodic stationary solution of (1.1), and let $u_{\mathrm{ini}} \in v+L^{1}\left(\mathbb{R}^{N}\right)$. Let $u$ be the unique solution of (1.1) with initial condition $u_{\mid t=0}=u_{\mathrm{ini}}$. Set

$$
M:=\int_{\mathbb{R}}\left(u_{\mathrm{ini}}-v\right) d y .
$$

Then as $t \rightarrow \infty$,

$$
\int_{\mathbb{R}^{N}}\left|u(t, y)-v(y)-\frac{1}{(1+2 t)^{N / 2}} f_{0}(y) F_{M}\left(\frac{y-c t}{\sqrt{1+2 t}}\right)\right| d y \rightarrow 0 .
$$

Remark 1.1. In fact, the regularity assumptions on the flux $A$ are not as stringent as stated in the theorem above. In particular, the conditions on the derivatives with respect to the space variable $y$ can be considerably reduced. When looking closely at the proof, the correct regularity assumptions on $A$ are

$$
\partial_{p}^{k} A \in L_{\mathrm{loc}}^{\infty}\left(\mathbb{T}^{N} \times \mathbb{R}\right)^{N} \quad \forall k \in\{0,1, \ldots, 4\}, \quad \operatorname{div}_{y} A, \operatorname{div}_{y} \partial_{p}^{4} A \in L_{\mathrm{loc}}^{\infty}\left(\mathbb{T}^{N} \times \mathbb{R}\right) .
$$

Remark 1.2. Notice that even in the linear case, Theorem 1.1 does not yield any convergence rate, in contrast with (1.3). This is due to the method of proof, which relies on abstract arguments from dynamical systems theory. The convergence rate obtained by Blanchet, Dolbeault and Kowalczyk in [5] is based on more "constructive" techniques, namely entropy dissipation methods and log-Sobolev inequalities. The counterpart of such techniques lies in the necessity for assumption (1.2). We emphasize that such an assumption is not necessary here.

Remark 1.3. Theorem 1.1 was previously proved in [18] in the special case $N=1$ and $M=0$ by Valérie Le Blanc. The proof in [18] is much shorter than the one presented here, but is very specific to the one-dimensional case, since it relies on an estimate on the number of sign changes of $u-v$. Unfortunately, the methods used in [18] do not seem to be adaptable to the general case under consideration here.

Remark 1.4. Let us now make precise the link between brownian ratchets and equation (1.1) in the linear case. In [5], A. Blanchet, J. Dolbeault and M. Kowalczyk study the long time behaviour of the solution $f=f(t, y)$ of the equation

$$
\partial_{t} f=\Delta_{y} f+\operatorname{div}_{y}(\nabla \psi(y-\omega t) f), \quad t>0, y \in \mathbb{R}^{N},
$$

with $\psi \in \mathcal{C}^{2}\left(\mathbb{T}^{N}\right), \omega \in \mathbb{R}^{N}$. Setting

$$
u(t, y)=f(t, y+\omega t) \quad \forall t>0, \forall y \in \mathbb{R}^{N},
$$

we see that $u$ satisfies

$$
\partial_{t} u+\operatorname{div}_{y}(\alpha(y) u)-\Delta_{y} u=0,
$$


where the drift coefficient $\alpha$ is given by

$$
\alpha(y)=-\omega-\nabla_{y} \psi(y) .
$$

Hence the study of (1.9) and that of (1.1) in the linear case are closely related; they are strictly equivalent in dimension one, since any function $\alpha \in \mathcal{C}^{1}(\mathbb{T})$ can be decomposed as

$$
\alpha=\int_{\mathbb{T}} \alpha+\left(\alpha-\int_{\mathbb{T}} \alpha\right)=\int_{\mathbb{T}} \alpha+\partial_{y} \phi, \quad \text { for some } \phi \in \mathcal{C}^{2}(\mathbb{T}) .
$$

The equivalence does not hold when $N \geq 2$, but in fact, all the results of [5] remain true for an arbitrary drift $\alpha \in \mathcal{C}^{1}\left(\mathbb{T}^{N}\right)$ (using exactly the same techniques as the ones developed in [5]). This will be a consequence of the analysis we will perform in the next sections. The choice for a function $\alpha$ with the structure (1.10) stems from physical considerations (see [4]): equation (1.9) describes the evolution of the density of particles in a travelling potential, moving with constant speed $\omega$.

In the course of the proof of Theorem 1.1, we will also prove that condition (1.2) holds for a large class of initial data. The precise result is the following:

Proposition 1.1. Assume that the flux $A$ is linear, and that $u_{\mathrm{ini}} \in v+L^{1}\left(\mathbb{R}^{N}\right)$ is such that

$$
\exists m>N+8, \quad \int_{\mathbb{R}^{N}}\left|u_{\text {ini }}(y)-v(y)\right|^{2}\left(1+|y|^{2}\right)^{m / 2} d y<\infty .
$$

Let $u$ be the unique solution of (1.1) with initial data $u_{\mathrm{ini}}$. Then (1.2) is satisfied. As a consequence (see [5]), (1.3) holds.

Hence for linear fluxes and for a large range of initial data, a rate of convergence can be given. The derivation of convergence rates in the nonlinear case goes beyond the scope of this article; in fact, the standard methods to derive convergence rates rely on the use of entropy-entropy dissipation inequalities (see [10] in the case of the Burgers equation), which we have chosen not to use here.

Another consequence of Theorem 1.1 is the stability of stationary shock profiles of equation (1.1) (see [8]) in dimension one:

Definition 1.1. Assume that $N=1$. A stationary shock profile is a stationary solution $U$ of (1.1) such that there exist periodic stationary solutions $v_{l}, v_{r}$ of (1.1) such that

$$
\lim _{y \rightarrow+\infty}\left[U(y)-v_{r}(y)\right]=0, \quad \lim _{y \rightarrow-\infty}\left[U(y)-v_{l}(y)\right]=0 .
$$

It was proved in [8] that the stability of shock profiles is a consequence of the stability of periodic stationary solutions. Thus we have the following

Corollary 1.1. Assume that $N=1$, and that the hypotheses of Theorem 1.1 are satisfied. Let $U \in L^{\infty}(\mathbb{R})$ be a stationary shock profile of (1.1). Let $u_{\mathrm{ini}} \in U+L^{1}(\mathbb{R})$ be such that

$$
\int_{\mathbb{R}}\left(u_{\text {ini }}-U\right)=0
$$


and let $u$ be the unique solution of (1.1) with initial data $u_{\mathrm{ini}}$. Then

$$
\lim _{t \rightarrow \infty}\|u(t)-U\|_{L^{1}(\mathbb{R})}=0 .
$$

The strategy of proof of Theorem 1.1 is close to the one developed in [12], in which M. Escobedo and E. Zuazua study the long time behaviour of a homogeneous version of (1.1). We also refer the interested reader to [11], in which M. Escobedo, J. L. Vazquez and E. Zuazua extend the analysis performed in [12] to the case when the flux has subcritical growth, and to [24], in which E. Zuazua extends the results of [12] to more general situations. The first step of the analysis consists in a self-similar change of variables, which helps us to focus on the appropriate length scales; this will be done in the next section, in which we also derive the equations on the limit profiles $f_{0}$ and $F_{M}$. Then, in Section 3, we obtain some compactness on the rescaled sequence by deriving some uniform $L^{2}$ bounds in weighted spaces. Eventually, we conclude the proof in Section 4 by using semigroup arguments inherited from dynamical systems theory.

Throughout the article, we will use the following notation: if $\psi \in L_{\mathrm{loc}}^{\infty}\left(\mathbb{R}^{N}\right)$, we set, for all $p \in[1, \infty)$,

$$
\begin{gathered}
L^{p}(\psi):=\left\{u \in L_{\mathrm{loc}}^{p}\left(\mathbb{R}^{N}\right): \int_{\mathbb{R}^{N}}|u|^{p} \psi<\infty\right\}, \quad\|u\|_{L^{p}(\psi)}=\left(\int_{\mathbb{R}^{N}}|u|^{p} \psi\right)^{1 / p}, \\
H^{1}(\psi):=\left\{u \in L^{2}(\psi), \nabla u \in L^{2}(\psi)\right\}, \quad\|u\|_{H^{1}(\psi)}^{2}=\|u\|_{L^{2}(\psi)}^{2}+\|\nabla u\|_{L^{2}(\psi)}^{2} .
\end{gathered}
$$

Sobolev spaces of the type $W^{s, p}(\psi), H^{s}(\psi)$, with $s \in \mathbb{N}$ arbitrary and $p \in[1, \infty)$, are defined in a similar fashion. When we write $\|u\|_{L^{p}\left(\mathbb{R}^{N}\right)}$, without specifying a weight function, we always refer to the usual $L^{p}$ norm in $\mathbb{R}^{N}$ with respect to the Lebesgue measure (i.e. $\psi \equiv 1$ ).

\section{The homogenized system}

The goal of this section is to analyze the expected asymptotic behaviour of the solution $u(t)$ of equation (1.1); to that end, we change the space and time variables and introduce a parabolic scaling, which is appropriate for the study of the long time behaviour of diffusion equations. Then, using a two-scale Ansatz in space and time which was introduced in [5], we construct an approximate solution of the rescaled system. Eventually, we recall and derive several properties of the limit system.

\subsection{Parabolic scaling}

Consider the solution $u \in L_{\text {loc }}^{\infty}\left([0, \infty) \times \mathbb{R}^{N}\right)$ of $(1.1)$, with $u_{\mid t=0}=u_{\text {ini }} \in v+L^{1} \cap$ $L^{\infty}\left(\mathbb{R}^{N}\right)$. It is a classical feature of scalar conservation laws that the semigroup associated with (1.1) is contractive in $L^{1}\left(\mathbb{R}^{N}\right)$. Hence, for all $t \geq 0, u(t) \in v+L^{1}\left(\mathbb{R}^{N}\right)$, and

$$
\|u(t)-v\|_{L^{1}\left(\mathbb{R}^{N}\right)} \leq\left\|u_{\mathrm{ini}}-v\right\|_{L^{1}\left(\mathbb{R}^{N}\right)} .
$$


Thus it is natural to compute the equation satisfied by $f(t)=u(t)-v \in L^{1}\left(\mathbb{R}^{N}\right)$ : since $v$ is a stationary solution of (1.1), we have

$$
\partial_{t} f+\operatorname{div}_{y} B(y, f)-\Delta_{y} f=0, \quad t>0, y \in \mathbb{R}^{N},
$$

where the flux $B$ is defined by

$$
B(y, f)=A(y, v(y)+f)-A(y, v(y)) \quad \forall(y, f) \in \mathbb{T}^{N} \times \mathbb{R} .
$$

The flux $B(y, f)$ vanishes at $f=0$, for all $y$. Moreover, if the flux $A$ satisfies the assumptions of Theorem 1.1, there exist $\alpha_{1} \in \mathcal{C}^{1}\left(\mathbb{T}^{N}\right)$ and $\tilde{B}_{1} \in \mathcal{C}\left(\mathbb{T}^{N} \times \mathbb{R}\right)$ such that

$$
B(y, f)=\alpha_{1}(y) f+\tilde{B}_{1}(y, f),
$$

and the flux $\tilde{B}_{1}$ is such that

$$
\forall X>0, \exists C_{X}>0, \forall f \in[-X, X], \forall y \in \mathbb{T}^{N}, \quad\left|\tilde{B}_{1}(y, f)\right| \leq C_{X}|f|^{2} .
$$

At some point in the proof, we will need a more refined approximation of $B$ in a neighbourhood of $f=0$; we thus also introduce $\alpha_{2}, \alpha_{3} \in L^{\infty}\left(\mathbb{T}^{N}\right)$ and $\tilde{B}_{3} \in L^{\infty}\left(\mathbb{T}^{N} \times \mathbb{R}\right)$ such that

$$
B(y, f)=\alpha_{1}(y) f+\alpha_{2}(y) f^{2}+\alpha_{3}(y) f^{3}+\tilde{B}_{3}(y, f),
$$

and the flux $\tilde{B}_{3}$ is such that for all $X>0$, there exists a constant $C_{X}>0$ such that for all $f \in[-X, X]$ and $y \in \mathbb{T}^{N}$,

$$
\left|\tilde{B}_{3}(y, f)\right| \leq C_{X}|f|^{4}, \quad\left|\operatorname{div}_{y} \tilde{B}_{3}(y, f)\right| \leq C_{X}|f|^{4}, \quad\left|\partial_{f} \tilde{B}_{3}(y, f)\right| \leq C_{X}|f|^{3} .
$$

The existence of $\alpha_{i}(i=1,2,3)$ and the bounds on $\tilde{B}_{1}, \tilde{B}_{3}$ are ensured by the assumption that $A \in W^{5, \infty}\left(\mathbb{T}^{N} \times \mathbb{R}\right)$. Notice in particular that

$$
\alpha_{1}(y)=\partial_{f} B(y, f)_{\mid f=0}=\left(\partial_{p} A\right)(y, v(y)) \quad \forall y \in \mathbb{T}^{N} .
$$

As explained in [5], the interplay between the diffusion and the drift $\alpha_{1}$ induces a displacement of the centre of mass. In the linear case, that is, when $\tilde{B}_{1}=0$, the evolution of the centre of mass can be computed as follows: since the function $f$ satisfies

$$
\partial_{t} f+\operatorname{div}_{y}\left(\alpha_{1} f\right)-\Delta_{y} f=0,
$$

we have

$$
\frac{d}{d t} \int_{\mathbb{R}^{N}} y f(t, y) d y=\int_{\mathbb{R}^{N}} \alpha_{1}(y) f(t, y) d y .
$$

Now, for $t \geq 0$ and $y \in \mathbb{T}^{N}$, set

$$
\tilde{f}(t, y)=\sum_{k \in \mathbb{Z}^{N}} f(t, y+k) .
$$

Since the function $\alpha_{1}$ is periodic, $\tilde{f}$ satisfies

$$
\partial_{t} \tilde{f}+\operatorname{div}_{y}\left(\alpha_{1} \tilde{f}\right)-\Delta_{y} \tilde{f}=0, \quad t>0, y \in \mathbb{T}^{N},
$$


and we have, for all $t \geq 0$,

$$
\int_{\mathbb{R}^{N}} \alpha_{1} f(t)=\int_{\mathbb{T}^{N}} \alpha_{1} \tilde{f}(t) .
$$

Using Lemma 1.1 of [19] together with a Poincaré inequality on the torus $\mathbb{T}^{N}$, it can be easily proved that as $t \rightarrow \infty, \tilde{f}(t)$ converges with exponential speed in $L^{1}\left(\mathbb{T}^{N}\right)$ towards $\langle\tilde{f}\rangle f_{0}$, where $f_{0}$ is the unique solution of (1.6). Additionally, notice that

$$
\langle\tilde{f}\rangle=\sum_{k \in \mathbb{Z}^{N}}\langle f(\cdot+k)\rangle=\int_{\mathbb{R}^{N}} f=M .
$$

Consequently, setting

$$
c:=\left\langle\alpha_{1} f_{0}\right\rangle
$$

we infer that in the linear case,

$$
\frac{d}{d t} \int_{\mathbb{R}}(y-c t) f \rightarrow 0 \quad \text { exponentially fast. }
$$

In fact, it turns out that the nonlinearity has no effect on this displacement, although this is not quite clear if we try to include the quadratic term $\tilde{B}_{1}$ in the above calculation. We will justify this result by formal calculations in the next subsection. Nonetheless, it can be proved in the case $N=1$ (see for instance [8]) that when $\left\|f_{0}\right\|_{1}$ is not too large,

$$
\|f(t)\|_{L^{2}(\mathbb{R})} \leq C \frac{\left\|f_{0}\right\|_{1}}{t^{1 / 4}} \quad \forall t>0,
$$

and more generally, the $L^{p}$ norm of $f(t)$ vanishes for long times for all $p \in(1, \infty]$. This somehow explains why the quadratic term does not modify the motion of the centre of mass for large times: the term $\tilde{B}_{1}(\cdot, f(t, \cdot))$ vanishes in $L^{1}(\mathbb{R})$ as $t \rightarrow \infty$. Hence, hereinafter, we choose to make in the general case the same change of variables as the one dictated by the linear case. Precisely, let $U \in L_{\mathrm{loc}}^{\infty}\left([0, \infty) \times \mathbb{R}^{N}\right)$ be such that

$$
f(t, y)=\frac{1}{(1+2 t)^{N / 2}} U\left(\log \sqrt{1+2 t}, \frac{y-c t}{\sqrt{1+2 t}}\right), \quad t \geq 0, y \in \mathbb{R}^{N} .
$$

This change of variables is classical in the study of long-time parabolic dynamics (see for instance [12]). In the present case, our change of variables is exactly the same as in [5]; straightforward calculations lead to

$$
\partial_{\tau} U-\operatorname{div}_{x}(x U)+R \operatorname{div}_{x}\left(\left(\alpha_{1}(z)-c\right) U\right)-\Delta_{x} U=-R^{N+1} \operatorname{div}_{x} \tilde{B}_{1}\left(z, U / R^{N}\right),
$$

with $\tau>0, x \in \mathbb{R}^{N}$, and where

$$
R=e^{\tau} \quad \text { and } \quad z=R x+c \frac{R^{2}-1}{2} .
$$

Studying the long time behaviour of $f$ amounts to studying the long time behaviour of $U$. Now, as $\tau \rightarrow \infty$, the quantity $R$ becomes very large, and thus the variable $z$ is highly 
oscillating. Hence, as emphasized in [5], the asymptotic study of equation (2.3) somehow falls into the scope of homogenization theory; the small parameter measuring the period of the oscillations is then $\varepsilon=R^{-1}=e^{-\tau}$. However, one substantial difference with classical homogenization problems is that the small parameter depends on time, which sometimes makes the proofs much more technical. We refer to [5] for more details.

Let us also mention that the homogenization of equation (2.3) with a "fixed" small parameter, and when the quadratic flux $\tilde{B}_{1}$ vanishes, has been performed by Thierry Goudon and Frédéric Poupaud in [14]. As a consequence, the formal asymptotic expansions which will be performed in the next section are in fact very close to the ones of [14].

\subsection{Formal derivation of the limit system}

As usual in homogenization problems (see [3] for instance), the idea is now to assume that the solution $U$ of (2.3) admits an asymptotic development in powers of the small parameter measuring the period of the oscillations; in the present case, the small parameter is $e^{-\tau}$, so that we expect the approximation to be valid for large times only. Hence, assume that when $\tau \gg 1$,

$$
U(\tau, x) \approx U_{0}(\tau, x, z)+e^{-\tau} U_{1}(\tau, x, z)+e^{-2 \tau} U_{2}(\tau, x, z)+\cdots
$$

where $z=e^{\tau} x+c\left(e^{2 \tau}-1\right) / 2$ stands for the fast variable and where for all $(\tau, x) \in$ $\mathbb{R}_{+} \times \mathbb{R}^{N}$, the function

$$
z \mapsto U_{i}(\tau, x, z)
$$

is $\mathbb{T}^{N}$-periodic. Plugging the Ansatz (2.4) into equation (2.3) and identifying the powers of $R=e^{\tau}$ leads to a cascade of equations on the terms $U_{0}, U_{1}$, etc. Notice that according to Lemma A.1 in the Appendix, $f \in L^{\infty}\left([0, \infty) \times \mathbb{R}^{N}\right)$, and thus $U / R^{N}$ is bounded in $L^{\infty}$.

- Terms of order $R^{2}$ : Identifying the highest order terms in equation (2.3) when $U$ is given by (2.4) leads to

$$
c \cdot \nabla_{z} U_{0}+\operatorname{div}_{z}\left(\left(\alpha_{1}-c\right) U_{0}\right)-\Delta_{z} U_{0}=-\Delta_{z} U_{0}+\operatorname{div}_{z}\left(\alpha_{1} U_{0}\right)=0, \quad z \in \mathbb{T}^{N} .
$$

We recall the following result, which is a straightforward consequence of the KreinRutman Theorem (see [9]):

Lemma 2.1. Let $\alpha \in L^{\infty}\left(\mathbb{T}^{N}\right)^{N}$. Consider the vector space

$$
E[\alpha]:=\left\{w \in H^{1}\left(\mathbb{T}^{N}\right):-\Delta_{z} w+\operatorname{div}_{z}(\alpha w)=0\right\} .
$$

Then $\operatorname{dim} E[\alpha]=1$, and there exists a unique function $m \in E[\alpha]$ such that $\langle m\rangle=1$. Moreover, $m \in W^{1, p}\left(\mathbb{T}^{N}\right)$ for all $p<\infty$, and

$$
\inf _{z \in \mathbb{T}^{N}} m>0
$$


In the present case, $E\left[\alpha_{1}\right]=\mathbb{R} f_{0}$, where $f_{0}$ is defined by (1.6). Hence there exists a function $F=F(\tau, x)$ such that

$$
U_{0}(\tau, x, z)=f_{0}(z) F(\tau, x) \quad \forall(\tau, x, z) \in[0, \infty) \times \mathbb{R}^{N} \times \mathbb{T}^{N} .
$$

- Terms of order $R^{1}$ : Concerning the terms of order $R^{1}=e^{\tau}$, the case when the space dimension is equal to one has to be treated separately. Indeed,

$$
\begin{aligned}
R^{N+1} \operatorname{div}_{x} \tilde{B}_{1}\left(z, U / R^{N}\right)= & R^{1-N} \operatorname{div}_{x}\left(\alpha_{2} U^{2}\right)+R^{1-2 N} \operatorname{div}_{x}\left(\alpha_{3} U^{3}\right) \\
& +R^{N+1} \operatorname{div}_{x}\left(\tilde{B}_{3}\left(z, U / R^{N}\right)\right),
\end{aligned}
$$

and using the bounds on $\tilde{B}_{3}$,

$$
\begin{aligned}
R^{N+1} \operatorname{div}_{x}\left(\tilde{B}_{3}\left(z, U / R^{N}\right)\right) & =R^{N}\left(\operatorname{div}_{z} \tilde{B}_{3}\right)\left(z, U / R^{N}\right)+R \nabla_{x} U \cdot\left(\partial_{U} \tilde{B}_{3}\right)\left(z, U / R^{N}\right) \\
& =\mathcal{O}\left(R^{-3 N}\right)+\mathcal{O}\left(R^{-3 N+2}\right)=\mathcal{O}\left(R^{-1}\right) .
\end{aligned}
$$

We infer that if $U$ is given by (2.4), then

$$
\begin{aligned}
R^{N+1} \operatorname{div}_{x} \tilde{B}_{1}\left(z, U / R^{N}\right)= & R^{2-N} \operatorname{div}_{z}\left(\alpha_{2} U_{0}^{2}\right) \\
& +R^{1-N}\left[\operatorname{div}_{x}\left(\alpha_{2} U_{0}^{2}\right)+2 \operatorname{div}_{z}\left(\alpha_{2} U_{0} U_{1}\right)\right] \\
& +R^{2-2 N} \operatorname{div}_{z}\left(\alpha_{3} U_{0}^{3}\right)+\mathcal{O}\left(R^{-1}\right) .
\end{aligned}
$$

Consequently, when $N \geq 2$, the term $U_{1}$ solves the equation

$$
-\Delta_{z} U_{1}+\operatorname{div}_{z}\left(\alpha_{1} U_{1}\right)=-\operatorname{div}_{x}\left(\left(\alpha_{1}-c\right) U_{0}\right)+2 \sum_{i=1}^{N} \frac{\partial^{2} U_{0}}{\partial x_{i} \partial z_{i}} .
$$

Since $U_{0}(t, x, z)=f_{0}(z) F(t, x)$, we have

$$
\left\langle\left(\alpha_{1}-c\right) U_{0}(t, x, \cdot)\right\rangle=F(t, x)\left(\left\langle\alpha_{1} f_{0}\right\rangle-c\right)=0
$$

by the definition of $c$. Hence the right-hand side of (2.7) has zero mean value, and the compatibility condition is satisfied. Thus for all $(t, x) \in[0, \infty) \times \mathbb{R}^{N},(2.7)$ has a unique solution in $H^{1}\left(\mathbb{T}^{N}\right)$. Moreover, using the linearity of (2.7) together with the expression (2.5), we infer that $U_{1}$ can be written as

$$
U_{1}(t, x, z)=f_{1}(z) \cdot \nabla_{x} F(t, x),
$$

where $f_{1} \in H^{1}\left(\mathbb{T}^{N}\right)^{N}$ satisfies

$$
-\Delta_{z} f_{1, i}+\operatorname{div}_{z}\left(\alpha_{1} f_{1, i}\right)=-f_{0}\left(\alpha_{1, i}-c_{i}\right)+2 \partial_{z_{i}} f_{0}, \quad \forall i \in\{1, \ldots, N\} .
$$

Notice that according to the regularity assumptions on the flux $A$, the function $\operatorname{div}_{y} \alpha_{1}$ belongs to $L^{\infty}\left(\mathbb{T}^{N}\right)$. Using boot-strap arguments for equation (1.6), we deduce that $f_{0} \in$ $W^{2, p}\left(\mathbb{T}^{N}\right)$ for all $p<\infty$, and therefore $f_{1} \in W^{2, p}\left(\mathbb{T}^{N}\right)$ for all $p<\infty$. In particular, $f_{1}, f_{0} \in W^{1, \infty}\left(\mathbb{T}^{N}\right)$. 
If $N=1$, on the other hand, the corrector $U_{1}$ solves the equation

$$
-\partial_{z z} U_{1}+\partial_{z}\left(\alpha_{1} U_{1}\right)=-\partial_{x}\left(\left(\alpha_{1}-c\right) U_{0}\right)+2 \frac{\partial^{2} U_{0}}{\partial x \partial z}-\partial_{z}\left(\alpha_{2} U_{0}^{2}\right) .
$$

Notice that the compatibility condition is satisfied, for the same reason as before. Hence in this case,

$$
U_{1}(t, x, z)=f_{1}(z) \partial_{x} F(t, x)+g_{1}(z) F(t, x)^{2},
$$

where $g_{1} \in H^{1}(\mathbb{T})$ solves

$$
-\Delta_{z} g_{1}+\partial_{z}\left(\alpha_{1} g_{1}\right)=-\partial_{z}\left(\alpha_{2}\left(f_{0}\right)^{2}\right)
$$

The fact that the compatibility condition is satisfied in all cases justifies a posteriori the use of the change of variables (2.2) in the nonlinear case. This means that, at least on a formal level, the displacement of the centre of mass of the function $f$ is unaffected by the presence of the quadratic term $\tilde{B}_{1}$.

- Terms of order $R^{0}$ : As we identify the terms of order one in equation (2.3), we obtain

$$
\begin{aligned}
& -\Delta_{z} U_{2}+\operatorname{div}\left(\alpha_{1} U_{2}\right) \\
& =-\partial_{\tau} U_{0}+\operatorname{div}_{x}\left(x U_{0}\right)+\Delta_{x} U_{0}-\operatorname{div}_{x}\left(\left(\alpha_{1}-c\right) U_{1}\right)+2 \sum_{i=1}^{N} \frac{\partial^{2} U_{1}}{\partial x_{i} \partial z_{i}}-\mathcal{A}_{N L},
\end{aligned}
$$

where the term $\mathcal{A}_{N L}$ stems from the expansion of the nonlinear term $\tilde{B}_{1}$. According to (2.6), we have

$$
\begin{aligned}
\mathcal{A}_{N L}=\partial_{x}\left(\alpha_{2} U_{0}^{2}\right)+2 \partial_{z}\left(\alpha_{2} U_{0} U_{1}\right)+\partial_{z}\left(\alpha_{3} U_{0}^{3}\right) & \text { if } N=1, \\
\mathcal{A}_{N L}=\operatorname{div}_{z}\left(\alpha_{2} U_{0}^{2}\right) & \text { if } N=2, \\
\mathcal{A}_{N L}=0 & \text { if } N \geq 3 .
\end{aligned}
$$

The evolution equation for the function $F$ follows from the compatibility condition; precisely, we obtain

$$
\partial_{\tau} F-\operatorname{div}_{x}(x F)-\Delta_{x} F+\operatorname{div}_{x}\left\langle\left(\alpha_{1}-c\right) U_{1}\right\rangle+\left\langle\mathcal{A}_{N L}\right\rangle=0 .
$$

We now distinguish between the cases $N \geq 2$ and $N=1$.

$\triangleright$ If $N \geq 2,\left\langle\mathcal{A}_{N L}\right\rangle=0$; using (2.8), we infer that $F$ satisfies

$$
\partial_{\tau} F-\operatorname{div}_{x}(x F)-\sum_{1 \leq i, j \leq N} \eta_{i, j} \frac{\partial^{2} F}{\partial x_{i} \partial x_{j}}=0, \quad \tau>0, x \in \mathbb{R}^{N} \text { with } N \geq 2,
$$

where the coefficients $\left(\eta_{i, j}\right)_{1 \leq i, j \leq N}$ are given by

$$
\eta_{i, j}=\delta_{i, j}-\left\langle\left(\alpha_{1, i}-c_{i}\right) f_{1, j}\right\rangle .
$$

The following lemma entails that equation (2.13) is well-posed: 
Lemma 2.2. The matrix $\eta:=\left(\eta_{i, j}\right)_{1 \leq i, j \leq N}$ is coercive.

Lemma 2.2 is proved in [14] for any $N \geq 1$, and its proof is recalled in [5] when $N=1$. For the reader's convenience, we sketch the main steps of the proof here, and we refer to [14, Proposition 4.6] for details.

Proof. Let $L$ be the differential operator $L \phi=-\Delta_{z} \phi+\operatorname{div}_{z}\left(\alpha_{1} \phi\right)$. The idea is to introduce, for all $j \in\{1, \ldots, N\}$, the function $\chi_{j}$ which solves the adjoint problem

$$
L^{*} \chi_{j}=-\Delta_{z} \chi_{j}-\alpha_{1} \cdot \nabla_{z} \chi_{j}=\alpha_{1, j}-c_{j}, \quad\left\langle\chi_{j}\right\rangle=0 .
$$

Since the right-hand side satisfies $\left\langle\left(\alpha_{1, j}-c_{j}\right) \psi\right\rangle=0$ for all $\psi \in \operatorname{ker} L=E\left[\alpha_{1}\right]=\mathbb{R} f_{0}$, the function $\chi_{j}$ is well-defined. For all $\xi \in \mathbb{R}^{N}$, we have

$$
\begin{aligned}
\sum_{i, j}\left\langle\left(\alpha_{1, i}-c_{i}\right) f_{1, j}\right\rangle \xi_{i} \xi_{j} & =\left\langle L^{*}(\chi \cdot \xi) f_{1} \cdot \xi\right\rangle=\left\langle\chi \cdot \xi\left(-f_{0}\left(\alpha_{1}-c\right) \cdot \xi+2 \nabla_{z} f_{0} \cdot \xi\right)\right\rangle \\
& =-\left\langle f_{0} \chi \cdot \xi L^{*}(\chi \cdot \xi)\right\rangle+\left\langle 2 \chi \cdot \xi \nabla_{z} f_{0} \cdot \xi\right\rangle \\
& =-\left\langle L\left(f_{0} \chi \cdot \xi\right) \chi \cdot \xi\right\rangle-2\left\langle f_{0} \xi \cdot \nabla_{z}(\chi \cdot \xi)\right\rangle .
\end{aligned}
$$

Expanding $L\left(f_{0} \chi \cdot \xi\right)$ and using the identity $L f_{0}=0$ leads to

$$
\left\langle L\left(f_{0} \chi \cdot \xi\right) \chi \cdot \xi\right\rangle=\left\langle f_{0}\left|\nabla_{z}(\chi \cdot \xi)\right|^{2}\right\rangle .
$$

Hence

$$
\sum_{1 \leq i, j \leq N} \eta_{i, j} \xi_{i} \xi_{j}=|\xi|^{2}+\left\langle f_{0}\left|\nabla_{z}(\chi \cdot \xi)\right|^{2}\right\rangle+2\left\langle f_{0} \xi \cdot \nabla_{z}(\chi \cdot \xi)\right\rangle=\left\langle f_{0}\left|\xi+\nabla_{z}(\chi \cdot \xi)\right|^{2}\right\rangle
$$

We deduce that

$$
\sum_{1 \leq i, j \leq N} \eta_{i, j} \xi_{i} \xi_{j} \geq 0 \quad \forall \xi \in \mathbb{R}^{N}
$$

Now, let $\xi \in \mathbb{R}^{N}$ be such that $\sum \eta_{i, j} \xi_{i} \xi_{j}=0$. Since $f_{0}(z)>0$ for all $z$, we infer that

$$
\xi+\nabla_{z}(\chi \cdot \xi)=0 \quad \forall z \in \mathbb{T}^{N} .
$$

Taking the average of the above inequality on $\mathbb{T}^{N}$ leads to $\xi=0$. Hence the matrix $\left(\eta_{i, j}\right)$ is coercive.

$\triangleright$ If $N=1$, we have

$$
\left\langle\mathcal{A}_{N L}\right\rangle=\partial_{x}\left\langle\alpha_{2} U_{0}^{2}\right\rangle=\left\langle\alpha_{2} f_{0}^{2}\right\rangle \partial_{x} F^{2} .
$$

Moreover, in this case $U_{1}$ is given by (2.11); hence

$$
\left\langle\operatorname{div}_{x}\left(\left(\alpha_{1}-c\right) U_{1}\right)\right\rangle=\left\langle\left(\alpha_{1}-c\right) f_{1}\right\rangle \partial_{x x} F+\left\langle\left(\alpha_{1}-c\right) g_{1}\right\rangle \partial_{x} F^{2} .
$$

Consequently, the compatibility condition reads

$$
\partial_{\tau} F-\partial_{x}(x F)+a \partial_{x} F^{2}-\eta \partial_{x x} F=0, \quad \tau>0, x \in \mathbb{R},
$$

where the coefficients $a, \eta$ are given by

$$
a:=\left\langle\alpha_{2} f_{0}^{2}\right\rangle+\left\langle\left(\alpha_{1}-c\right) g_{1}\right\rangle, \quad \eta:=1-\left\langle\left(\alpha_{1}-c\right) f_{1}\right\rangle .
$$

Lemma 2.2 states that the diffusion coefficient $\eta$ is positive. 
Remark 2.1. (i) The fact that the coefficient $a$ appearing in (1.8) is zero for all $N \geq 2$ is consistent with the results of M. Escobedo and E. Zuazua in [12]: indeed, the authors of [12] consider the convection-diffusion equation

$$
v_{t}-\Delta v=\alpha \cdot \nabla\left(|v|^{q-1} v\right) \quad \text { in }(0, \infty) \times \mathbb{R}^{N}
$$

for some exponent $q \geq 1+1 / N$ and for $\alpha \in \mathbb{R}^{N}$. They prove that if $q>1+1 / N$, the asymptotic behaviour of $v$ is given by the heat kernel, i.e. the convective term does not play any role. On the contrary, if $q=1+1 / N, v$ behaves asymptotically like the self-similar solution of a nonlinear equation.

In the present case, because of the Taylor expansion (2.1), the flux $B$ consists of a linear drift term and a quadratic term. Hence the study performed in this paper is related to the case $q=2$ of [12]; we infer that $q=1+1 / N$ if and only if $N=1$, and we check that this is the only case when a nonlinear behaviour can be observed at the limit.

(ii) In order to have a better understanding of the large time behaviour of equation (1.1), it would be interesting to compute the next term of the development, in the spirit of the paper by E. Zuazua [23]. In particular, the nonlinear behaviour of the equation is expected to appear at the next order for all $N \in \mathbb{N}$. Notice that this implies that the term $U_{1}$ is not, in fact, the next term in the development. However, such an analysis is beyond the scope of the present article.

The formal derivation of an approximate solution is now complete. In the following subsections, we recall or prove several results concerning the well-posedness and the long time behaviour of equations (2.13) and (2.15). We will often refer to the equation on $F$ as the "homogenized equation"; this term refers to equation (2.13) when $N \geq 2$, and (2.15) when $N=1$.

\subsection{Existence and uniqueness of stationary solutions of the homogenized equation}

This subsection is concerned with the existence and uniqueness (in suitable function spaces) of stationary solutions of the homogenized equations (2.13) and (2.15). In the case when $N=1$, or when $\left(\eta_{i, j}\right)_{1 \leq i, j \leq N}=\lambda I$ for some $\lambda>0$, such results are stated in [1]. In the general case, we merely use a linear change of variables, and the problem is then reduced to the case of an isotropic diffusion.

Lemma 2.3. Assume that $N \geq 2$. For $\gamma>0$, set $\psi_{\gamma}: x \in \mathbb{R}^{N} \mapsto \exp \left(\gamma|x|^{2}\right)$. Then there exists $\gamma>0$ such that for all $M \in \mathbb{R}$, there exists a unique function $F_{M} \in H^{1}\left(\psi_{\gamma}\right)$ satisfying

$$
-\sum_{1 \leq i, j \leq N} \eta_{i, j} \partial_{i} \partial_{j} F_{M}-\operatorname{div}_{x}\left(x F_{M}\right)=0, \quad \int_{\mathbb{R}^{N}} F_{M}=M .
$$

Furthermore, the following properties hold:

(i) for all $M \in \mathbb{R}, F_{M}=M F_{1}$;

(ii) $F_{1} \in W^{2, p} \cap \mathcal{C}^{\infty}\left(\mathbb{R}^{N}\right)$ for all $p \in[1, \infty)$, and $F_{1} \in H^{2}\left(\psi_{\gamma}\right)$;

(iii) $F_{1}(x)>0$ for all $x \in \mathbb{R}^{N}$. 
Proof. The idea is to perform an affine change of variables in order to transform the diffusion term into a laplacian. Precisely, set

$$
s_{i, j}=\frac{\eta_{i, j}+\eta_{j, i}}{2}, \quad 1 \leq i, j \leq N .
$$

Then the matrix $S=\left(s_{i, j}\right)$ is symmetric and positive definite (see Lemma 2.2); hence there exists an orthogonal matrix $O \in \mathcal{M}_{n}(\mathbb{R})$ and positive numbers $\lambda_{j}$ such that

$$
S=O^{T} \operatorname{Diag}\left(\lambda_{1}, \ldots, \lambda_{N}\right) O .
$$

Let us change the variables by setting

$$
x=P y \quad \text { with } \quad P:=O^{T} \operatorname{Diag}\left(\lambda_{1}^{1 / 2}, \ldots, \lambda_{N}^{1 / 2}\right),
$$

and for any function $F \in L^{1}\left(\mathbb{R}^{N}\right)$, define

$$
\tilde{F}(y)=F(P y) .
$$

It can be readily checked that for all $x \in \mathbb{R}^{N}$,

$$
\sum_{i, j} \eta_{i, j} \frac{\partial^{2} F}{\partial x_{i} \partial x_{j}}(x)=\sum_{k, l} \tilde{\eta}_{k, l} \frac{\partial^{2} \tilde{F}}{\partial y_{k} \partial y_{l}}\left(P^{-1} x\right),
$$

where the coefficients $\tilde{\eta}_{k, l}$ are given by

$$
\tilde{\eta}_{k, l}=\sum_{i, j}\left(P^{-1}\right)_{k, i}\left(P^{-1}\right)_{l, j} s_{i, j}=\left(P^{-1} S\left(P^{-1}\right)^{T}\right)_{k, l} .
$$

Using the definitions of the matrices $P$ and $S$, we infer that

$$
\tilde{\eta}=P^{-1} S\left(P^{-1}\right)^{T}=I_{N} .
$$

Thus the diffusion term is transformed into a laplacian with this change of variables.

Let us now compute the drift term. We have

$$
x_{i}=(P y)_{i}
$$

and, denoting by $\left(e_{1}, \ldots, e_{N}\right)$ the canonical basis of $\mathbb{R}^{N}$,

$$
\frac{\partial F(x)}{\partial x_{i}}=\frac{\partial}{\partial x_{i}} \tilde{F}\left(P^{-1} x\right)=\left(P^{-1} e_{i}\right) \cdot \nabla_{y} \tilde{F}\left(P^{-1} x\right) .
$$

Thus, always setting $x=P y$,

$$
\begin{aligned}
x \cdot \nabla_{x} F(x) & =\sum_{i=1}^{N}(P y)_{i}\left(P^{-1} e_{i}\right) \cdot \nabla_{y} \tilde{F}(y)=\left[P^{-1}\left(\sum_{i=1}^{N}(P y)_{i} e_{i}\right)\right] \cdot \nabla_{y} \tilde{F}(y) \\
& =\left(P^{-1} P y\right) \cdot \nabla_{y} \tilde{F}(y)=y \cdot \nabla_{y} \tilde{F}(y) .
\end{aligned}
$$


Notice that this property is in fact independent of the definition of the matrix $P$. Consequently, $F_{M}$ is a solution of (2.17) if and only if $\tilde{F}_{M}$ satisfies

$$
-\Delta_{y} \tilde{F}_{M}-\operatorname{div}_{y}\left(y \tilde{F}_{M}\right)=0, \quad \int_{\mathbb{R}^{N}} \tilde{F}_{M}=(\operatorname{det} S)^{-1 / 2} M .
$$

The only solutions of the above equation in $H^{1}\left(\mathbb{R}^{N}\right)$ are Gaussian functions. Hence there exists a unique solution of (2.17) in $H^{1}\left(\mathbb{R}^{N}\right)$ for all $M$, and this solution is given by

$$
F_{M}(x)=C M \exp \left(-\frac{1}{2}\left|P^{-1} x\right|^{2}\right),
$$

where the positive constant $C$ is a normalization factor. Moreover,

$$
\left|P^{-1} x\right|^{2}=\left|\operatorname{Diag}\left(\lambda_{1}^{-1 / 2}, \ldots, \lambda_{N}^{-1 / 2}\right) O x\right|^{2},
$$

and thus, since $|O x|^{2}=|x|^{2}$,

$$
\left(\max _{1 \leq i \leq N} \lambda_{i}\right)^{-1}|x|^{2} \leq\left|P^{-1} x\right|^{2} \leq\left(\min _{1 \leq i \leq N} \lambda_{i}\right)^{-1}|x|^{2}
$$

All the properties of the lemma follow, with

$$
\gamma<\left(2 \max _{1 \leq i \leq N} \lambda_{i}\right)^{-1} .
$$

In the case when $N=1$, the existence of a stationary solution is treated in [1]. Hence we merely recall the main results of [1] in that regard.

Lemma 2.4 (Aguirre, Escobedo, Zuazua). Let $M \in \mathbb{R}$ be arbitrary, and let $a \in \mathbb{R}$ and $\eta>0$. Let $\gamma:=(2 \eta)^{-1}$. Then there exists a unique function $F_{M} \in H^{1}\left(\psi_{\gamma}\right)$ which satisfies

$$
-\eta \partial_{x x} F_{M}-\partial_{x}\left(x F_{M}\right)+a \partial_{x} F_{M}^{2}=0, \quad \int_{\mathbb{R}} F_{M}=M .
$$

Moreover, $F_{M}$ enjoys the following properties:

(i) $F_{M} \in W^{2, p} \cap \mathcal{C}^{\infty}(\mathbb{R})$ for all $p \in[1, \infty)$, and $F_{M} \in H^{2}\left(\psi_{\gamma}\right)$;

(ii) if $M>0$, then $F_{M}(x)>0$ for all $x \in \mathbb{R}^{N}$.

We deduce from the above lemma that if $\gamma^{\prime}<\gamma$, then there exists a constant $C_{\gamma^{\prime}}$ such that

$$
\left|F_{M}(x)\right|,\left|\partial_{x} F_{M}(x)\right| \leq C_{\gamma^{\prime}} \exp \left(-\gamma^{\prime} x^{2}\right) \quad \forall x \in \mathbb{R} .
$$

Indeed, since $F_{M} \in H^{2}\left(\psi_{\gamma}\right)$, it can be easily proved that $F_{M} \psi_{\gamma^{\prime}} \in H^{2}(\mathbb{R})$ for all $\gamma^{\prime}<\gamma$. Sobolev embeddings then imply that $F_{M} \psi_{\gamma^{\prime}} \in W^{1, \infty}(\mathbb{R})$.

The existence of stationary solutions of (2.13) and (2.15) is thus ensured. We now tackle the study of the properties of equations (2.13) and (2.15), focusing in particular on the long time behaviour and on regularity issues. 


\subsection{Long time behaviour of the homogenized equation}

We begin with a definition of the weight function $K \in \mathcal{C}^{\infty}\left(\mathbb{R}^{N}\right)$, which plays a central role in the theory of existence. For $N \geq 2$, we use the change of variables (2.18), which was introduced in the proof of Lemma 2.3. This allows us to transform the matrix $\left(\eta_{i, j}\right)$ into the identity matrix. For $t>0, y \in \mathbb{R}^{N}$, set $\tilde{F}(t, y)=F(t, P y)$. If $F$ is a solution of (2.13), then $\tilde{F}$ solves

$$
\partial_{t} \tilde{F}-\operatorname{div}_{y}(y \tilde{F})-\Delta_{y} \tilde{F}=0 .
$$

Consequently, the results of [12] can be directly applied to $\tilde{F}$, for which existence is proved in the function space $L^{2}\left(K_{0}\right)$, where $K_{0}(y)=\exp \left(y^{2} / 2\right)$. Performing the inverse change of variables, it is clear that the relevant weight function is given by

$$
K(x):=K_{0}\left(P^{-1} x\right)=\exp \left(\left|P^{-1} x\right|^{2} / 2\right) .
$$

Notice that by the definition of the matrix $P$, there exist positive constants $\gamma, \gamma^{\prime}$ such that

$$
\exp \left(\gamma^{\prime} x^{2}\right) \leq K(x) \leq \exp \left(\gamma x^{2}\right) \quad \forall x \in \mathbb{R}^{N} .
$$

When $N=1$, the weight function $K$ is given by

$$
K(x):=\exp \left(\frac{|x|^{2}}{2 \eta}\right) .
$$

We immediately deduce from [12] the following proposition:

Proposition 2.1. Let $F_{\mathrm{ini}} \in L^{\infty}\left(\mathbb{R}^{N}\right) \cap L^{2}(K)$. Then the homogenized problem has a unique solution

$$
F \in \mathcal{C}\left([0, \infty), L^{2}(K)\right) \cap \mathcal{C}\left((0, \infty), H^{2}(K)\right) \cap \mathcal{C}^{1}\left((0, \infty), L^{2}(K)\right)
$$

such that $F_{\mid t=0}=F_{\text {ini. }}$. Moreover,

$$
\lim _{t \rightarrow \infty}\left\|F(t)-F_{M}\right\|_{L^{1}\left(\mathbb{R}^{N}\right)}=0,
$$

where $F_{M}$ is the unique stationary solution of the homogenized problem with mass $M=$ $\int_{\mathbb{R}^{N}} F_{\text {ini. }}$.

Consequently, the homogenized equations (2.15) and (2.13) are well-posed. We conclude this section by stating a result on the construction of an approximate solution:

Definition 2.1. Let $F \in \mathcal{C}\left([0, \infty), L^{2}(K)\right) \cap \mathcal{C}\left((0, \infty), H^{2}(K)\right)$. We define the approximate solution of (2.3) associated with $F$ by

$$
U^{\mathrm{app}}[F](\tau, x ; R)=U_{0}(\tau, x, z)+R^{-1} U_{1}(\tau, x, z)+R^{-2} V_{2}(\tau, x, z),
$$

with $\tau \geq 0, x \in \mathbb{R}^{N}, R>0$ and $z:=R x+c\left(R^{2}-1\right) / 2$, and where

- $U_{0}$ is defined by (2.5);

- $U_{1}$ is defined by (2.8) if $N \geq 2$ and by (2.11) if $N=1$; 
- $V_{2}$ is defined by

$$
\begin{aligned}
-\Delta_{z} V_{2}+\operatorname{div}\left(\alpha_{1} V_{2}\right)= & \left(f_{0}(z)-1\right)\left[-\partial_{\tau} F+\operatorname{div}_{x}(x F)+\Delta_{x} F\right] \\
& +\left\langle\operatorname{div}_{x}\left(\left(\alpha_{1}-c\right) U_{1}\right)\right\rangle-\operatorname{div}_{x}\left(\left(\alpha_{1}-c\right) U_{1}\right) \\
& +2 \sum_{i=1}^{N} \frac{\partial^{2} U_{1}}{\partial x_{i} \partial z_{i}}-\mathcal{A}_{N L}+\left\langle\mathcal{A}_{N L}\right\rangle .
\end{aligned}
$$

Notice that we do not require, in the above definition, that $F$ is a solution of (2.13) or (2.15); hence the right-hand side in the equation on the term of order $R^{-2}$ is slightly modified, so that the compatibility condition is satisfied and $V_{2}$ is well-defined. Of course, if $F$ is a solution of (2.15) or (2.13), the equation on $V_{2}$ becomes (2.12) and $V_{2}=U_{2}$.

We then have the following result:

Lemma 2.5. (i) Let $M \in \mathbb{R}$ be arbitrary. Define the function $U \in L^{\infty}\left([0, \infty) \times \mathbb{R}^{N}\right)$ ) by

$$
U(\tau, x):=U^{\mathrm{app}}\left[F_{M}\right]\left(\tau, x ; e^{\tau}\right) .
$$

Then $U$ is a solution of

$$
\begin{aligned}
\partial_{\tau} U-\operatorname{div}_{x}(x U)-\Delta_{x} U+R \operatorname{div}_{x}\left(\left(\alpha_{1}(z)-c\right) U\right) \\
=-R^{N+1} \operatorname{div}_{x} \tilde{B}_{1}\left(z, U / R^{N}\right)+U^{\mathrm{rem}},
\end{aligned}
$$

where the remainder term $U^{\mathrm{rem}}$ is such that there exist $C>0, \gamma>0$ such that

$$
\left\|U^{\mathrm{rem}}(\tau)\right\|_{L^{1}\left(e^{\gamma|x|^{2}}\right)}+\left\|U^{\mathrm{rem}}(\tau)\right\|_{L^{\infty}\left(\mathbb{R}^{N}\right)} \leq C e^{-\tau} \quad \forall \tau \geq 0 .
$$

(ii) Let $F_{\text {ini }} \in L^{\infty}\left(\mathbb{R}^{N}\right) \cap L^{2}(K)$, and let $F \in \mathcal{C}\left([0, \infty), L^{2}(K)\right)$ be the unique solution of the homogenized equation such that $F_{\mid t=0}=F_{\text {ini. }}$. Let $\rho \in \mathcal{C}_{0}^{\infty}\left(\mathbb{R}^{N}\right)$ be a mollifying kernel $\left(\rho \geq 0, \int \rho=1\right)$, and let $F_{\delta}:=F *_{x} \rho_{\delta}$, where $\rho_{\delta}=\delta^{-N} \rho(\cdot / \delta)$, for $\delta>0$. Let $\left(\tau_{n}\right)_{n \geq 0}$ be a sequence of positive numbers such that $\lim _{n \rightarrow \infty} \tau_{n}=+\infty$. For $n \in \mathbb{N}$ and $\delta>0$, define the function $u_{n}^{\delta}$ by

$$
u_{n}^{\delta}(\tau, x)=U^{\mathrm{app}}\left[F_{\delta}\right]\left(\tau, x ; e^{\tau_{n}+\tau}\right), \quad x \in \mathbb{R}^{N}, \tau \geq 0 .
$$

Then $u_{n}^{\delta}$ satisfies, with $R_{n}=e^{\tau_{n}+\tau}$ and $z_{n}=R_{n} x+c\left(R_{n}^{2}-1\right) / 2$,

$$
\begin{aligned}
\partial_{\tau} u_{n}^{\delta}-\operatorname{div}_{x}\left(x u_{n}^{\delta}\right)+R_{n} \operatorname{div}_{x}\left(\left(\alpha_{1}\left(z_{n}\right)-c\right) u_{n}^{\delta}\right)-\Delta_{x} u_{n}^{\delta} \\
=-R_{n}^{N+1} \operatorname{div}_{x} \tilde{B}_{1}\left(z, u_{n}^{\delta} / R_{n}^{N}\right)+r_{n}^{\delta},
\end{aligned}
$$

where the remainder term $r_{n}^{\delta}$ satisfies, for all $T>0$,

$$
\left\|r_{n}^{\delta}\right\|_{L^{\infty}\left([0, T], L^{1}\left(\mathbb{R}^{N}\right)\right)} \leq \omega_{T}(\delta)+C_{\delta, T} e^{-\tau_{n}},
$$

where $\omega_{T}: \mathbb{R}_{+} \rightarrow \mathbb{R}_{+}$is a function depending only on $T$ such that $\lim _{\delta \rightarrow 0^{+}} \omega_{T}(\delta)$ $=0$.

The proof of the above lemma follows the calculations of the first section; the proof is lengthy but straightforward, and is therefore left to the reader. The fact that $U^{\text {rem }}$ has exponential decay in $x$ is a consequence of Lemmas 2.3 and 2.4. 


\section{Weighted $L^{2}$ bounds for the rescaled equation}

As explained in the previous section, we choose to work with the rescaled equation (2.3) rather than with the original one (1.1). In fact, it can be easily checked that Theorem 1.1 is equivalent to the following proposition:

Proposition 3.1. Let $U_{\mathrm{ini}} \in L^{1}\left(\mathbb{R}^{N}\right)$, and let $M:=\int_{\mathbb{R}^{N}} U_{\text {ini. Let }} U \in \mathcal{C}\left([0, \infty), L^{1}\left(\mathbb{R}^{N}\right)\right)$ be the unique solution of (2.3) with initial data $U_{\mid \tau=0}=U_{\text {ini }}$. Then

$$
\lim _{\tau \rightarrow \infty} \int_{\mathbb{R}^{N}}\left|U(\tau, x)-f_{0}\left(e^{\tau} x+c \frac{e^{2 \tau}-1}{2}\right) F_{M}(x)\right| d x=0,
$$

where the velocity $c$ is defined by (1.7), and $F_{M} \in L^{1}\left(\mathbb{R}^{N}\right)$ is the unique stationary solution of the homogenized equation (2.13), (2.15) with total mass $M$.

In turn, since the function $f_{0} \in L^{\infty}\left(\mathbb{T}^{N}\right)$ is such that $\inf _{\mathbb{T}^{N}} f_{0}>0$, the above statement is equivalent to

$$
\lim _{\tau \rightarrow \infty}\left\|V(\tau)-F_{M}\right\|_{L^{1}\left(\mathbb{R}^{N}\right)}=0
$$

where the function $V=V(\tau, x)$ is defined by

$$
V(\tau, x):=\frac{U(\tau, x)}{f_{0}\left(e^{\tau} x+c \frac{e^{2 \tau}-1}{2}\right)}, \quad \tau \geq 0, x \in \mathbb{R}^{N} .
$$

The proof of Proposition 3.1 consists of essentially two steps: first, we prove compactness properties in $L^{1}\left(\mathbb{R}^{N}\right)$ for the family $(V(\tau))_{\tau \geq 0}$. To that end, we derive uniform bounds with respect to $\tau$ in weighted $L^{2}$ spaces; this step will be achieved in the current section. Then, using techniques inherited from dynamical systems theory, we prove in the next section that the limit of any converging sequence $V\left(\tau_{n}\right)$ is equal to $F_{M}$. As emphasized in the introduction, the proof of convergence relies on rather abstract arguments, and thus does not yield any rate of convergence in general. However, when the flux $A$ is linear, the weighted $L^{2}$ bounds allow us to prove that the family $U(\tau)$ has uniformly bounded moments of order four, and thus (1.2) holds. As proved in [5], the convergence stated in Theorem 1.1 then takes place with algebraic rate.

The main result of this section is the following:

Proposition 3.2. Let $U_{\text {ini }} \in L^{1} \cap L^{\infty}\left(\mathbb{R}^{N}\right)$, and let $U \in \mathcal{C}\left([0, \infty), L^{1}\left(\mathbb{R}^{N}\right)\right)$ be the unique solution of (2.3) with initial data $U_{\mid \tau=0}=U_{\text {ini }}$. Let $m>2(N+1)$ be arbitrary, and assume that

$$
\int_{\mathbb{R}^{N}}\left|U_{\text {ini }}(x)\right|^{2}\left(1+|x|^{2}\right)^{m / 2} d x<\infty .
$$

Then there exists a constant $C_{m}>0$ (depending only on $m, N$, and on the flux $A$ ) such that if $\left\|U_{\mathrm{ini}}\right\|_{L^{1}\left(\mathbb{R}^{N}\right)} \leq C_{m}$, then

$$
\begin{gathered}
\sup _{\tau \geq 0} \int_{\mathbb{R}^{N}}|V(\tau, x)|^{2}\left(1+|x|^{2}\right)^{m / 2} d x<\infty, \\
\sup _{\tau \geq 0} \int_{\tau}^{\tau+1} \int_{\mathbb{R}^{N}}\left|\nabla_{x} V(s, x)\right|^{2} d x d s<\infty .
\end{gathered}
$$


As a consequence, there exists a sequence $\left(\tau_{n}\right)$ of positive numbers such that $\tau_{n} \in$ $[n, n+1]$ for all $n$, and the sequence $\left(V\left(\tau_{n}, x\right)\right)_{n \geq 0}$ is compact in $L^{1}\left(\mathbb{R}^{N}\right)$. Moreover, if the flux $A$ is linear, then $C_{m}=+\infty$ for all $m>2(N+1)$.

Before proving the bounds (3.2), we explain how they entail the existence of a converging sequence. Thus we assume that (3.2) holds for the time being. First, for any $X \geq 1, \tau \geq 0$, we have

$$
\begin{aligned}
\int_{|x| \geq X} & |V(\tau, x)| d x \\
\leq & \left(\int_{|x| \geq X}|V(\tau, x)|^{2}\left(1+|x|^{2}\right)^{m / 2} d x\right)^{1 / 2}\left(\int_{|x| \geq X}\left(1+|x|^{2}\right)^{-m / 2} d x\right)^{1 / 2} \\
& \leq C X^{(N-m) / 2}\left(\sup _{\tau \geq 0} \int_{\mathbb{R}^{N}}|V(\tau, x)|^{2}\left(1+|x|^{2}\right)^{m / 2} d x\right)^{1 / 2} .
\end{aligned}
$$

Since $m>N$, we infer that the family $\{V(\tau, x)\}_{\tau \geq 0}$ is equi-integrable.

Moreover, let $\mathcal{K} \subset \mathbb{R}^{N}$ be an arbitrary compact set, and let $h \in \mathbb{R}^{N}$ be arbitrary, with $|h| \leq 1$. Let

$$
\tilde{\mathcal{K}}:=\left\{x \in \mathbb{R}^{N}: d(x, \mathcal{K}) \leq 1\right\} .
$$

The set $\tilde{\mathcal{K}}$ is clearly compact. Then

$$
\begin{aligned}
\int_{\mathcal{K}}|V(\tau, x+h)-V(\tau, x)| d x & \leq|h| \int_{\mathcal{K}} \int_{0}^{1}|\nabla V|(\tau, x+\lambda h) d \lambda d x \\
& \leq|h| \int_{\tilde{\mathcal{K}}}|\nabla V(\tau, z)| d z \\
& \leq|h||\tilde{\mathcal{K}}|^{1 / 2}\left(\int_{\mathbb{R}^{N}}\left|\nabla_{x} V(\tau, x)\right|^{2} d x\right)^{1 / 2} .
\end{aligned}
$$

Now, for all $n \in \mathbb{N}$, there exists $\tau_{n} \in[n, n+1]$ such that

$$
\int_{\mathbb{R}^{N}}\left|\nabla_{x} V\left(\tau_{n}, x\right)\right|^{2} d x \leq \int_{n}^{n+1} \int_{\mathbb{R}^{N}}\left|\nabla_{x} V(s, x)\right|^{2} d x d s .
$$

Consequently, there exists a constant $C$, depending only on $\mathcal{K}$ and on the bounds on $V$ in $L_{\mathrm{loc}}^{2}\left([0, \infty), H^{1}\right)$, such that

$$
\forall n \in \mathbb{N}, \quad \int_{\mathcal{K}}\left|V\left(\tau_{n}, x+h\right)-V\left(\tau_{n}, x\right)\right| d x \leq C|h| .
$$

Hence the sequence $\left\{V\left(\tau_{n}, x\right)\right\}_{n \geq 0}$ is equicontinuous in $L^{1}\left(\mathbb{R}^{N}\right)$.

Notice also that

$$
\sup _{n \geq 0}\left\|V\left(\tau_{n}\right)\right\|_{L^{1}\left(\mathbb{R}^{N}\right)} \leq \frac{1}{\inf _{\mathbb{T}^{N}} f_{0}} \sup _{n \geq 0}\left\|U\left(\tau_{n}\right)\right\|_{L^{1}\left(\mathbb{R}^{N}\right)} \leq \frac{\left\|U_{\text {ini }}\right\|_{L^{1}\left(\mathbb{R}^{N}\right)}}{\inf _{\mathbb{T}^{N}} f_{0}} .
$$

Thus the sequence $\left\{V\left(\tau_{n}, x\right)\right\}_{n \geq 0}$ is bounded in $L^{1}\left(\mathbb{R}^{N}\right)$. 
According to classical results of functional analysis (see for instance [6]), we infer that the sequence $\left(V\left(\tau_{n}\right)\right)_{n \geq 0}$ is compact in $L^{1}$.

The rest of the section is devoted to the proof of the bounds (3.2). We first prove that $V \in L_{\text {loc }}^{\infty}\left([0, \infty), L^{2}\left(\left(1+|x|^{2}\right)^{m / 2}\right)\right)$. Then, using the construction of approximate solutions of (2.3) performed in the previous section, we derive an energy inequality on the function $V$. Carefully controlling the nonlinear terms appearing in this energy inequality, we are led to (3.2).

Before addressing the proof, we recall a result which will play a key role in several arguments: since $U_{\text {ini }} \in L^{\infty} \cap L^{1}\left(\mathbb{R}^{N}\right)$, there exists a positive constant $C$, depending only on the flux $A$ and on $\left\|U_{\text {ini }}\right\|_{1},\left\|U_{\text {ini }}\right\|_{\infty}$, such that

$$
\|U(\tau)\|_{L^{\infty}\left(\mathbb{R}^{N}\right)} \leq C e^{N \tau} .
$$

Indeed, performing backwards the parabolic scaling (2.2), it turns out that this inequality is equivalent to the boundedness of $u$ in $L^{\infty}\left([0, \infty) \times \mathbb{R}^{N}\right)$, where $u$ is the solution of (1.1) with initial data $v+U_{\text {ini }}$. And the $L^{\infty}$ bound on $u$ follows from Lemma A. 1 in the Appendix.

First step: The family $V(\tau)$ is locally bounded in $L^{2}\left(\left(1+|x|^{2}\right)^{m / 2}\right)$. This amounts in fact to proving that $U \in L_{\text {loc }}^{\infty}\left([0, \infty), L^{2}\left(\left(1+|x|^{2}\right)^{m / 2}\right)\right)$. Hence, multiply (2.3) by $U(\tau, x)\left(1+|x|^{2}\right)^{m / 2}$ and integrate with respect to the variable $x$. Always with the notation $R=e^{\tau}, z=R x+c\left(R^{2}-1\right) / 2$, this leads to

$$
\begin{aligned}
\frac{1}{2} \frac{d}{d \tau} & \int_{\mathbb{R}^{N}}|U(\tau, x)|^{2}\left(1+|x|^{2}\right)^{m / 2} d x \\
= & -\int_{\mathbb{R}^{N}}\left|\nabla_{x} U\right|^{2}\left(1+|x|^{2}\right)^{m / 2} d x-m \int_{\mathbb{R}^{N}}\left(x \cdot \nabla_{x} U\right) U\left(1+|x|^{2}\right)^{-1+m / 2} d x \\
& -\frac{1}{2} \int_{\mathbb{R}^{N}}\left(x \cdot \nabla_{x}|U|^{2}\right)\left(1+|x|^{2}\right)^{m / 2} d x-m \int_{\mathbb{R}^{N}}|U|^{2}|x|^{2}\left(1+|x|^{2}\right)^{-1+m / 2} d x \\
& -R^{N+1} \int_{\mathbb{R}^{N}}\left[B\left(z, \frac{U(\tau, x)}{R^{N}}\right)-c \frac{U(\tau, x)}{R^{N}}\right] \cdot \nabla_{x} U\left(1+|x|^{2}\right)^{m / 2} d y \\
& -m R^{N+1} \int_{\mathbb{R}^{N}}\left[B\left(z, \frac{U(\tau, x)}{R^{N}}\right)-c \frac{U(\tau, x)}{R^{N}}\right] \cdot x U\left(1+|x|^{2}\right)^{-1+m / 2} d x .
\end{aligned}
$$

Since $U(\tau) / R^{N}$ is bounded (see (3.3)), there exists a constant $C$ such that

$$
\left|B\left(R x+c \frac{R^{2}-1}{2}, \frac{U(\tau, x)}{R^{N}}\right)-c \frac{U(\tau, x)}{R^{N}}\right| \leq C \frac{|U(\tau, x)|}{R^{N}} .
$$

Moreover,

$$
\left|x\left(1+|x|^{2}\right)^{-1+m / 2}\right|,\left.|| x\right|^{2}\left(1+|x|^{2}\right)^{-1+m / 2} \mid \leq\left(1+|x|^{2}\right)^{m / 2} \quad \forall x \in \mathbb{R}^{N} .
$$

Hence, using the Cauchy-Schwarz inequality, we infer that the last two terms in (3.4) are bounded by

$$
\frac{1}{4} \int_{\mathbb{R}^{N}}\left|\nabla_{x} U\right|^{2}\left(1+|x|^{2}\right)^{m / 2} d x+C R^{2} \int_{\mathbb{R}^{N}}|U|^{2}\left(1+|x|^{2}\right)^{m / 2} d x .
$$


On the other hand,

$$
\begin{aligned}
\mid \int_{\mathbb{R}^{N}}\left(x \cdot \nabla_{x}|U|^{2}\right)(1 & \left.+|x|^{2}\right)^{m / 2} d x \mid \\
& =\left.\left|\int_{\mathbb{R}^{N}}\right| U\right|^{2}\left(N\left(1+|x|^{2}\right)^{m / 2}+m x^{2}\left(1+|x|^{2}\right)^{-1+m / 2}\right) d x \mid \\
& \leq C \int_{\mathbb{R}^{N}}|U|^{2}\left(1+|x|^{2}\right)^{m / 2} d x
\end{aligned}
$$

Gathering all the terms, we deduce that there exist $c \in(0,1)$ and $C>0$ such that

$$
\begin{aligned}
& \frac{d}{d \tau} \int_{\mathbb{R}^{N}}|U(\tau, x)|^{2}\left(1+|x|^{2}\right)^{m / 2} d x \\
& \quad \leq-c \int_{\mathbb{R}^{N}}\left|\nabla_{x} U(\tau, x)\right|^{2}\left(1+|x|^{2}\right)^{m / 2} d x+C e^{2 \tau} \int_{\mathbb{R}^{N}}|U(\tau, x)|^{2}\left(1+|x|^{2}\right)^{m / 2} d x .
\end{aligned}
$$

Using Gronwall's Lemma, we infer that

$$
U \in L_{\mathrm{loc}}^{\infty}\left([0, \infty), L^{2}\left(\left(1+|x|^{2}\right)^{m / 2}\right)\right), \quad \nabla_{x} U \in L_{\mathrm{loc}}^{2}\left([0, \infty), L^{2}\left(\left(1+|x|^{2}\right)^{m / 2}\right)\right) .
$$

Second step: The energy inequality. The idea here is the following: assume momentarily that the flux $B$ is linear, that is, $\tilde{B}_{1}=0$. Let $\psi \in L^{\infty}\left([0, \infty) \times \mathbb{R}^{N}\right)$ be a solution of (2.3) such that $\psi(\tau, x)>0$ for all $\tau, x$. Then, according to [19], for any convex function $H \in \mathcal{C}^{2}(\mathbb{R})$, we have

$$
\frac{d}{d \tau} \int_{\mathbb{R}^{N}} \psi(\tau, x) H\left(\frac{U(\tau, x)}{\psi(\tau, x)}\right) d x=-\int_{\mathbb{R}^{N}} H^{\prime \prime}\left(\frac{U(\tau, x)}{\psi(\tau, x)}\right)\left|\nabla_{x}\left(\frac{U(\tau, x)}{\psi(\tau, x)}\right)\right|^{2} d x .
$$

Taking $H: x \in \mathbb{R} \mapsto x^{2}$, we infer that

$$
\sup _{\tau \geq 0} \int_{\mathbb{R}^{N}}|U(\tau, x)|^{2} \frac{d x}{\psi(\tau, x)}<\infty .
$$

Hence, if $\psi(\tau, x)$ behaves like $\left(1+|x|^{2}\right)^{-m / 2}$ for $|x|$ large, the $L^{2}$ bound in (3.2) is proved.

Thus the goal of this step is to build a positive function $\tilde{U}$ which behaves like $\left(1+|x|^{2}\right)^{-m / 2}$ for $|x|$ large, and which is an approximate solution of the linear part of (2.3), with remainder terms of order one. Using calculations similar to the ones in [19], we then derive an inequality on the energy

$$
\int_{\mathbb{R}^{N}}\left|\frac{U(\tau, x)}{\tilde{U}(\tau, x)}\right|^{2} \tilde{U}(\tau, x) d x .
$$

From now on, we no longer assume that $\tilde{B}_{1}=0$.

The definition of $\tilde{U}$ is inspired from the construction of an approximate solution in the previous section. Precisely, we set

$\tilde{U}(\tau, x)=f_{0}(z) h_{m}(x)+e^{-\tau} f_{1}(z) \cdot \nabla_{x} h_{m}(x), \quad \tau \geq 0, x \in \mathbb{R}^{N}, z=e^{\tau} x+c \frac{e^{2 \tau}-1}{2}$, 
where the function $f_{1} \in W^{1, \infty}\left(\mathbb{T}^{N}\right)^{N}$ is defined by (2.9), and where

$$
h_{m}(x):=\left(1+|x|^{2}\right)^{-m / 2} .
$$

Notice that even when $N=1$, the structure of the function $\tilde{U}$ is inspired from the linear case: there is no term of the form $h_{m}^{2}$ in $\tilde{U}$. The nonlinear term in the flux $B$ will be treated independently.

Remember that $\inf _{\mathbb{T}^{N}} f_{0}>0$; since

$$
\nabla_{x} h_{m}(x)=-m \frac{x}{1+|x|^{2}} h_{m}, \quad x \in \mathbb{R}^{N},
$$

we deduce that there exists $\tau_{0}>0$ (depending on $m$ ) such that

$$
0<\frac{1}{2} f_{0}(z) h_{m}(x) \leq \tilde{U}(\tau, x) \leq 2 f_{0}(z) h_{m}(x) \quad \forall x \in \mathbb{R}^{N}, \tau \geq \tau_{0} .
$$

We now compute, for $\tau \geq \tau_{0}$, the rate of growth (or decay) of the energy $\int|U|^{2} \tilde{U}^{-1}$. Using equation (2.3) and performing several integrations by parts, we obtain

$$
\begin{aligned}
\frac{d}{d \tau} \int_{\mathbb{R}^{N}}\left|\frac{U(\tau, x)}{\tilde{U}(\tau, x)}\right|^{2} \tilde{U}(\tau, x) d x \\
=-2 \int_{\mathbb{R}^{N}}\left|\nabla_{x}\left(\frac{U(\tau, x)}{\tilde{U}(\tau, x)}\right)\right|^{2} \tilde{U}(\tau, x) d x \\
\quad+\int_{\mathbb{R}^{N}}\left|\frac{U(\tau, x)}{\tilde{U}(\tau, x)}\right|^{2}\left[-\partial_{\tau} \tilde{U}+\Delta_{x} \tilde{U}+\operatorname{div}_{x}(x \tilde{U})-e^{\tau} \operatorname{div}_{x}\left(\left(\alpha_{1}(z)-c\right) \tilde{U}\right)\right] d x \\
+2 e^{(N+1) \tau} \int_{\mathbb{R}^{N}} \tilde{B}_{1}\left(z, \frac{U(\tau, x)}{e^{N \tau}}\right) \cdot \nabla_{x}\left(\frac{U(\tau, x)}{\tilde{U}(\tau, x)}\right) d x
\end{aligned}
$$

By definition of $\tilde{U}$, we have

$$
\begin{aligned}
-\partial_{\tau} \tilde{U}+\Delta_{x} \tilde{U} & +\operatorname{div}_{x}(x \tilde{U})-e^{\tau} \operatorname{div}_{x}\left(\left(\alpha_{1}(z)-c\right) \tilde{U}\right) \\
= & \operatorname{div}_{x}\left(x h_{m}\right) f_{0}(z)+f_{0}(z) \Delta_{x} h_{m}(x)+2 \sum_{1 \leq i, j \leq N} \frac{\partial f_{1, i}}{\partial z_{j}}(z) \frac{\partial^{2} h_{m}(x)}{\partial x_{i} \partial x_{j}} \\
& -\sum_{1 \leq i, j \leq N}\left[\left(\alpha_{1, i}-c_{i}\right) f_{1, j}\right](z) \frac{\partial^{2} h_{m}(x)}{\partial x_{i} \partial x_{j}} \\
& +e^{-\tau} \sum_{1 \leq i, j \leq N} f_{1, i}(z)\left[\frac{\partial}{\partial x_{j}}\left(x_{j} \partial_{x_{i}} h_{m}(x)\right)+\frac{\partial^{3} h_{m}(x)}{\partial x_{i} \partial x_{j}^{2}}\right]
\end{aligned}
$$

where

$$
z=e^{\tau} x+c \frac{e^{2 \tau}-1}{2}
$$


Notice that

$$
\operatorname{div}_{x}\left(x h_{m}(x)\right)=(N-m) h_{m}(x)+\frac{m}{\left(1+|x|^{2}\right)^{1+m / 2}},
$$

and there exists a constant $C$ (depending on $m$ and $N$ ) such that for all $i, j \in\{1, \ldots, N\}$,

$$
\begin{gathered}
\left|\frac{\partial^{2} h_{m}(x)}{\partial x_{i} \partial x_{j}}\right| \leq C \frac{1}{\left(1+|x|^{2}\right)^{1+m / 2}}, \\
\left|\nabla_{x} h_{m}(x)\right|+|| x\left|\frac{\partial^{2} h_{m}(x)}{\partial x_{i} \partial x_{j}}\right|+\left|\frac{\partial^{3} h_{m}(x)}{\partial x_{i} \partial x_{j}^{2}}\right| \leq C h_{m}(x) .
\end{gathered}
$$

Remember that $N-m<0$ and that inequality (3.5) holds; hence we infer that for $\tau \geq \tau_{0}$ with $\tau_{0}$ large enough,

$$
\begin{aligned}
-\partial_{\tau} \tilde{U}+\Delta_{x} \tilde{U}+\operatorname{div}_{x}(x \tilde{U})-e^{\tau} \operatorname{div}_{x}\left(\left(\alpha_{1}(z)-c\right) \tilde{U}\right) \\
\quad \leq(N-m) f_{0}(z) h_{m}(x)+C e^{-\tau} h_{m}(x)+C \frac{1}{\left(1+|x|^{2}\right)^{1+m / 2}} \\
\quad \leq \frac{N-m}{4} \tilde{U}(\tau, x)+C \frac{1}{\left(1+|x|^{2}\right)^{1+m / 2}}
\end{aligned}
$$

On the other hand, since the flux $\tilde{B}_{1}$ is quadratic near the origin and $U / e^{N \tau}$ is bounded, we have

$$
\begin{aligned}
\mid e^{(N+1) \tau} \int_{\mathbb{R}^{N}} \tilde{B}_{1}\left(z, \frac{U(\tau, x)}{e^{N \tau}}\right) & \cdot \nabla_{x}\left(\frac{U(\tau, x)}{\tilde{U}(\tau, x)}\right) d x \mid \\
& \leq C e^{(1-N) \tau} \int_{\mathbb{R}^{N}}|U(\tau, x)|^{2}\left|\nabla_{x}\left(\frac{U(\tau, x)}{\tilde{U}(\tau, x)}\right)\right| d x
\end{aligned}
$$

Gathering all the terms, we obtain

$$
\begin{aligned}
\frac{d}{d \tau} \int_{\mathbb{R}^{N}}\left|\frac{U}{\tilde{U}}\right|^{2} \tilde{U}+\frac{m-N}{4} \int_{\mathbb{R}^{N}}\left|\frac{U}{\tilde{U}}\right|^{2} \tilde{U} & +2 \int_{\mathbb{R}^{N}}\left|\nabla\left(\frac{U}{\tilde{U}}\right)\right|^{2} \tilde{U} \\
\leq & C \int_{\mathbb{R}^{N}}\left(\frac{U(\cdot, x)}{\tilde{U}(\cdot, x)}\right)^{2} \frac{d x}{\left(1+|x|^{2}\right)^{1+m / 2}} \\
& +C e^{(1-N) \tau} \int_{\mathbb{R}^{N}}|U|^{2}\left|\nabla_{x}\left(\frac{U}{\tilde{U}}\right)\right|
\end{aligned}
$$

Notice that when the flux $A$ is linear, the term (3.8) is zero.

Third step: Control of the term (3.7). Set $\phi:=U / \tilde{U}$; then according to the first step,

$$
\phi \in L_{\mathrm{loc}}^{\infty}\left(\left[\tau_{0}, \infty\right), L^{2}\left(h_{m}\right)\right) \cap L_{\mathrm{loc}}^{2}\left(\left[\tau_{0}, \infty\right), H^{1}\left(h_{m}\right)\right) .
$$

Moreover,

$$
\nabla\left(\phi^{2} h_{m}\right)=2 \phi h_{m} \nabla \phi+\phi^{2} \nabla h_{m}
$$


since $\left|\nabla h_{m}\right| \leq m h_{m}$, we deduce that $\phi^{2} h_{m} \in L_{\text {loc }}^{1}\left(\left[\tau_{0}, \infty\right), W^{1,1}\left(\mathbb{R}^{N}\right)\right)$, and thus, using Sobolev embeddings, $\phi^{2} h_{m} \in L_{\text {loc }}^{1}\left(\left[\tau_{0}, \infty\right), L^{p^{*}}\left(\mathbb{R}^{N}\right)\right)$, where $p^{*}:=N /(N-1)$ if $N \geq 2$, and $p^{*}=+\infty$ if $N=1$. Additionally, the following inequality holds: according to the Gagliardo-Nirenberg-Sobolev inequality, there exists a constant $C$, depending only on $N$ and $m$, such that for all $\tau \geq \tau_{0}$,

$$
\begin{aligned}
\left\|\phi^{2} h_{m}(\tau)\right\|_{L^{p^{*}}\left(\mathbb{R}^{N}\right)} & \leq C\left\|\nabla\left(\phi^{2} h_{m}(\tau)\right)\right\|_{L^{1}\left(\mathbb{R}^{N}\right)} \\
& \leq C\|\phi(\tau)\|_{L^{2}\left(h_{m}\right)}\|\nabla \phi(\tau)\|_{L^{2}\left(h_{m}\right)}+C\|\phi(\tau)\|_{L^{2}\left(h_{m}\right)}^{2} .
\end{aligned}
$$

We use the above inequality in order to control the term (3.7). First, let us write

$$
\int_{\mathbb{R}^{N}}|\phi(\tau, x)|^{2}\left(1+|x|^{2}\right)^{-(1+m / 2)} d x=\int_{\mathbb{R}^{N}}\left(\phi^{2}(\tau) h_{m}\right)^{a}\left(|\phi(\tau)| h_{m}\right)^{b},
$$

where the exponents $a, b$ satisfy

$$
\left\{\begin{array}{l}
2 a+b=2 \\
a m / 2+b m / 2=1+m / 2
\end{array}\right.
$$

which leads to $a=1-2 / m, b=4 / m$. Notice that $a, b \in(0,1)$, provided $m$ is large enough ( $m>4$, which is always satisfied if $m>2(N+1))$.

Then, using Hölder's inequality, we infer

$$
\int_{\mathbb{R}^{N}}|\phi(\tau, x)|^{2}\left(1+|x|^{2}\right)^{-(1+m / 2)} d x \leq\left\|\phi^{2}(\tau) h_{m}\right\|_{L^{p}\left(\mathbb{R}^{N}\right)}^{a}\left\|\phi(\tau) h_{m}\right\|_{L^{1}\left(\mathbb{R}^{N}\right)}^{b},
$$

where the parameter $p$ is given by

$$
p=a(1-b)^{-1}=\frac{1-2 / m}{1-4 / m} .
$$

Notice that $p$ is always larger than one. In order to be able to interpolate $L^{p}$ between $L^{1}$ and $L^{p^{*}}, p$ must also be smaller than $p^{*}$; if $N=1, p^{*}=\infty$, and thus we always have $p<p^{*}$. If $N \geq 2$, this condition amounts to $m>2(N+1)$; we assume that $m$ always satisfies this assumption in what follows.

Now, let $\theta \in(0,1)$ such that

$$
\frac{1}{p}=\frac{\theta}{1}+\frac{1-\theta}{p^{*}}
$$

using once again Hölder's inequality, we obtain

$$
\begin{aligned}
\int_{\mathbb{R}^{N}}|\phi(\cdot, x)|^{2}\left(1+|x|^{2}\right)^{-(1+m / 2)} d x \leq & \left\|\phi^{2} h_{m}\right\|_{L^{1}\left(\mathbb{R}^{N}\right)}^{a \theta}\left\|\phi^{2} h_{m}\right\|_{L^{p^{*}}\left(\mathbb{R}^{N}\right)}^{a(1-\theta)}\left\|\phi h_{m}\right\|_{L^{1}\left(\mathbb{R}^{N}\right)}^{b} \\
\leq & C\left\|\phi^{2} h_{m}\right\|_{L^{1}\left(\mathbb{R}^{N}\right)}^{a \theta+a(1-\theta) / 2}\|\nabla \phi\|_{L^{2}\left(h_{m}\right)}^{a(1-\theta)}\left\|\phi h_{m}\right\|_{L^{1}\left(\mathbb{R}^{N}\right)}^{b} \\
& +C\left\|\phi^{2} h_{m}\right\|_{L^{1}\left(\mathbb{R}^{N}\right)}^{a}\left\|\phi h_{m}\right\|_{L^{1}\left(\mathbb{R}^{N}\right)}^{b}
\end{aligned}
$$


Straightforward computations lead to

$$
a \theta+a \frac{1-\theta}{2}=1-\frac{N+2}{m}, \quad a(1-\theta)=\frac{2 N}{m} .
$$

Hence, using Young's inequality, we deduce that for all $\lambda>0$, there exists a constant $C_{\lambda}$ such that

$$
\begin{aligned}
& \int_{\mathbb{R}^{N}}|\phi(\tau, x)|^{2}\left(1+|x|^{2}\right)^{-(1+m / 2)} d x \\
& \quad \leq \lambda\left\|\phi^{2}(\tau) h_{m}\right\|_{L^{1}\left(\mathbb{R}^{N}\right)}+\lambda\|\nabla \phi(\tau)\|_{L^{2}\left(h_{m}\right)}^{2}+C_{\lambda}\left\|\phi(\tau) h_{m}\right\|_{L^{1}\left(\mathbb{R}^{N}\right)}^{2} .
\end{aligned}
$$

Using inequality (3.5) and choosing the parameter $\lambda$ small enough leads eventually to

$$
\begin{aligned}
(3.7) \leq & \frac{m-N}{16} \int_{\mathbb{R}^{N}}\left|\frac{U(\tau)}{\tilde{U}(\tau)}\right|^{2} \tilde{U}(\tau)+\frac{1}{2} \int_{\mathbb{R}^{N}}\left|\nabla\left(\frac{U(\tau)}{\tilde{U}(\tau)}\right)\right|^{2} \tilde{U}(\tau) \\
& +C\left(\int_{\mathbb{R}^{N}}|U(\tau, x)| d x\right)^{2}
\end{aligned}
$$

for all $\tau \geq \tau_{0}$.

\section{Fourth step: Control of the term (3.8).}

Remark 3.1. We recall that $(3.8)=0$ if the flux $A$ is linear. Hence this step is required only in the nonlinear case.

Using inequality (3.3), we infer that there exists a constant $C$ such that

$$
(3.8) \leq C \int_{\mathbb{R}^{N}}|U(\tau, x)|^{1+1 / N}\left|\nabla_{x}\left(\frac{U(\tau, x)}{\tilde{U}(\tau, x)}\right)\right| d x .
$$

From now on, we treat the cases $N=1, N=2$, and $N \geq 3$ separately, and we set $\phi=U / \tilde{U}$.

- If $N=1$, we have, for all $\tau \geq \tau_{0}$,

$$
\begin{aligned}
\int_{\mathbb{R}^{N}}|U(\tau, x)|^{1+1 / N} \mid & \nabla_{x}\left(\frac{U(\tau, x)}{\tilde{U}(\tau, x)}\right) \mid d x \\
& \leq C \int_{\mathbb{R}}\left(\partial_{x} \phi(\tau) h_{m}^{1 / 2}\right)\left(\phi(\tau) h_{m}\right)^{1 / 2}\left(\phi^{3 / 2}(\tau) h_{m}\right) \\
& \leq C\left\|\partial_{x} \phi(\tau)\right\|_{L^{2}\left(h_{m}\right)}\|U(\tau)\|_{L^{1}(\mathbb{R})}^{1 / 2}\left\|\phi^{3 / 2}(\tau) h_{m}\right\|_{L^{\infty}(\mathbb{R})} \\
& \leq C\left\|\partial_{x} \phi(\tau)\right\|_{L^{2}\left(h_{m}\right)}\|U(\tau)\|_{L^{1}(\mathbb{R})}^{1 / 2}\left\|\partial_{x}\left(\phi^{3 / 2}(\tau) h_{m}\right)\right\|_{L^{1}(\mathbb{R})}
\end{aligned}
$$

Moreover,

$$
\partial_{x}\left(\phi^{3 / 2} h_{m}\right)=\frac{3}{2} \phi^{1 / 2} h_{m} \partial_{x} \phi+\phi^{3 / 2} \partial_{x} h_{m},
$$


and thus

$$
\left\|\partial_{x}\left(\phi^{3 / 2} h_{m}\right)\right\|_{L^{1}(\mathbb{R})} \leq C\left\|\phi h_{m}\right\|_{L^{1}}^{1 / 2}\left(\left\|\partial_{x} \phi\right\|_{L^{2}\left(h_{m}\right)}+\|\phi\|_{L^{2}\left(h_{m}\right)}\right) .
$$

Eventually, we obtain, using once again (3.5),

$$
(3.8) \leq C\|U(\tau)\|_{L^{1}(\mathbb{R})}\left[\int_{\mathbb{R}}\left|\nabla\left(\frac{U(\tau)}{\tilde{U}(\tau)}\right)\right|^{2} \tilde{U}(\tau)+\int_{\mathbb{R}}\left|\frac{U(\tau)}{\tilde{U}(\tau)}\right|^{2} \tilde{U}(\tau)\right] .
$$

Since

$$
\|U(\tau)\|_{L^{1}(\mathbb{R})} \leq\left\|U_{\text {ini }}\right\|_{L^{1}(\mathbb{R})} \quad \forall \tau \geq 0,
$$

we infer that if $\left\|U_{\text {ini }}\right\|_{L^{1}(\mathbb{R})}$ is sufficiently small, then

$$
(3.8) \leq \frac{m-N}{16} \int_{\mathbb{R}^{N}}\left|\frac{U(\tau)}{\tilde{U}(\tau)}\right|^{2} \tilde{U}(\tau)+\frac{1}{2} \int_{\mathbb{R}^{N}}\left|\nabla\left(\frac{U(\tau)}{\tilde{U}(\tau)}\right)\right|^{2} \tilde{U}(\tau) .
$$

- If $N=2$, using the Sobolev embedding $W^{1,1}\left(\mathbb{R}^{2}\right) \subset L^{2}\left(\mathbb{R}^{2}\right)$, we obtain, for $\tau \geq \tau_{0}$,

$$
\begin{aligned}
\int_{\mathbb{R}^{N}}|U(\cdot, x)|^{1+1 / N}\left|\nabla_{x}\left(\frac{U(\cdot, x)}{\tilde{U}(\cdot, x)}\right)\right| d x & \leq C \int_{\mathbb{R}^{2}}\left(|\phi|^{3 / 2} h_{m}\right)\left(\left|\nabla_{x} \phi\right| h_{m}^{1 / 2}\right) \\
& \leq C\left\||\phi|^{3 / 2} h_{m}\right\|_{L^{2}\left(\mathbb{R}^{2}\right)}\left\|\nabla_{x} \phi\right\|_{L^{2}\left(h_{m}\right)} \\
& \leq C\left\|\nabla\left(|\phi|^{3 / 2} h_{m}\right)\right\|_{L^{1}\left(\mathbb{R}^{2}\right)}\left\|\nabla_{x} \phi\right\|_{L^{2}\left(h_{m}\right)} .
\end{aligned}
$$

As is the case $N=1$, we have

$$
\left\|\nabla\left(|\phi|^{3 / 2} h_{m}\right)\right\|_{L^{1}\left(\mathbb{R}^{2}\right)} \leq C\left\|\phi h_{m}\right\|_{L^{1}\left(\mathbb{R}^{2}\right)}^{1 / 2}\left(\left\|\partial_{x} \phi\right\|_{L^{2}\left(h_{m}\right)}+\|\phi\|_{L^{2}\left(h_{m}\right)}\right) .
$$

Hence we are led to

$$
(3.8) \leq C\|U(\tau)\|_{L^{1}\left(\mathbb{R}^{2}\right)}^{1 / 2}\left[\int_{\mathbb{R}^{2}}\left|\nabla \frac{U(\tau)}{\tilde{U}(\tau)}\right|^{2} \tilde{U}(\tau)+\int_{\mathbb{R}^{2}}\left|\frac{U(\tau)}{\tilde{U}(\tau)}\right|^{2} \tilde{U}(\tau)\right] .
$$

Following exactly the same argument as in the case $N=1$, we deduce that if $\left\|U_{\text {ini }}\right\|_{L^{1}\left(\mathbb{R}^{2}\right)}$ is sufficiently small, then (3.11) holds.

- If $N \geq 3$, we have, for $\tau \geq \tau_{0}$,

$$
\begin{aligned}
\int_{\mathbb{R}^{N}}|U(\cdot, x)|^{1+1 / N}\left|\nabla_{x}\left(\frac{U(\cdot, x)}{\tilde{U}(\cdot, x)}\right)\right| d x & \leq C \int_{\mathbb{R}^{N}}|\phi|^{1+1 / N}\left|\nabla_{x} \phi\right| h_{m}^{1+1 / N} \\
& \leq C \int_{\mathbb{R}^{N}}\left(|\phi| h_{m}\right)^{1 / N}\left(\left|\nabla_{x} \phi\right| h_{m}^{1 / 2}\right)\left(|\phi| h_{m}^{1 / 2}\right) \\
& \leq C\left\|\phi h_{m}\right\|_{L^{1}\left(\mathbb{R}^{N}\right)}^{1 / N}\left\|\nabla_{x} \phi\right\|_{L^{2}\left(h_{m}\right)}\left\|\phi h_{m}^{1 / 2}\right\|_{L^{p}\left(\mathbb{R}^{N}\right)}
\end{aligned}
$$

where the parameter $p$ is such that

$$
\frac{1}{N}+\frac{1}{2}+\frac{1}{p}=1
$$


i.e. $p=2 N /(N-2)$. Using the Sobolev embedding $H^{1}\left(\mathbb{R}^{N}\right) \subset L^{p}\left(\mathbb{R}^{N}\right)$, we have

$$
\left\||\phi| h_{m}^{1 / 2}\right\|_{L^{p}\left(\mathbb{R}^{N}\right)} \leq C\left\|\nabla_{x}\left(|\phi| h_{m}^{1 / 2}\right)\right\|_{L^{2}\left(\mathbb{R}^{N}\right)} \leq C\left(\|\nabla \phi\|_{L^{2}\left(h_{m}\right)}+\|\phi\|_{L^{2}\left(h_{m}\right)}\right) .
$$

Thus, once again, we obtain

$$
(3.8) \leq C\|U(\tau)\|_{L^{1}\left(\mathbb{R}^{N}\right)}^{1 / N}\left[\int_{\mathbb{R}^{N}}\left|\nabla\left(\frac{U(\tau)}{\tilde{U}(\tau)}\right)\right|^{2} \tilde{U}(\tau)+\int_{\mathbb{R}^{2}}\left|\frac{U(\tau)}{\tilde{U}(\tau)}\right|^{2} \tilde{U}(\tau)\right],
$$

and thus (3.11) holds as long as $\left\|U_{\text {ini }}\right\|_{L^{1}\left(\mathbb{R}^{N}\right)}$ is not too large.

Gathering inequalities (3.6), (3.10) and (3.11), we infer that if $\left\|U_{\mathrm{ini}}\right\|_{L^{1}\left(\mathbb{R}^{N}\right)}$ is sufficiently small, then for all $\tau \geq \tau_{0}$,

$$
\begin{gathered}
\frac{d}{d \tau} \int_{\mathbb{R}^{N}}\left|\frac{U(\tau)}{\tilde{U}(\tau)}\right|^{2} \tilde{U}(\tau)+\frac{m-N}{8} \int_{\mathbb{R}^{N}}\left|\frac{U(\tau)}{\tilde{U}(\tau)}\right|^{2} \tilde{U}(\tau)+\int_{\mathbb{R}^{N}}\left|\nabla\left(\frac{U(\tau)}{\tilde{U}(\tau)}\right)\right|^{2} \tilde{U}(\tau) \\
\leq C\left(\int_{\mathbb{R}^{N}}|U(\tau, x)| d x\right)^{2} \leq C\left\|U_{\mathrm{ini}}\right\|_{L^{1}}^{2}\left(\mathbb{R}^{N}\right)
\end{gathered}
$$

Fifth step: Conclusion. Let $C_{1}:=(m-N) / 8$ and $C_{2}:=C\left\|U_{\text {ini }}\right\|_{L^{1}\left(\mathbb{R}^{N}\right)}^{2}$. Using a Gronwall type argument, we deduce that for all $\tau \geq \tau_{0}$, we have

$$
\begin{aligned}
& \int_{\mathbb{R}^{N}}\left|\frac{U(\tau)}{\tilde{U}(\tau)}\right|^{2} \tilde{U}(\tau)+\int_{\tau_{0}}^{\tau} e^{-C_{1}(\tau-s)}\left(\int_{\mathbb{R}^{N}}\left|\nabla\left(\frac{U(s)}{\tilde{U}(s)}\right)\right|^{2} \tilde{U}(s)\right) d s \\
& \quad \leq e^{-C_{1}\left(\tau-\tau_{0}\right)} \int_{\mathbb{R}^{N}}\left|\frac{U\left(\tau_{0}\right)}{\tilde{U}\left(\tau_{0}\right)}\right|^{2} \tilde{U}\left(\tau_{0}\right)+\frac{C_{2}}{C_{1}} \leq C \int_{\mathbb{R}^{N}}\left|U\left(\tau_{0}, x\right)\right|^{2}\left(1+|x|^{2}\right)^{m / 2} d x+\frac{C_{2}}{C_{1}} .
\end{aligned}
$$

Using (3.5), we infer

$$
\begin{gathered}
\sup _{\tau \geq \tau_{0}} \int_{\mathbb{R}^{N}}|U(\tau, x)|^{2}\left(1+|x|^{2}\right)^{m / 2} d x \leq C \int_{\mathbb{R}^{N}}\left|U\left(\tau_{0}, x\right)\right|^{2}\left(1+|x|^{2}\right)^{m / 2} d x+C \frac{C_{2}}{C_{1}}, \\
\sup _{\tau \geq \tau_{0}} \int_{\tau}^{\tau+1} \int_{\mathbb{R}^{N}}\left|\nabla\left(\frac{U(s)}{\tilde{U}(s)}\right)\right|^{2} h_{m} d s \leq C \int_{\mathbb{R}^{N}}\left|U\left(\tau_{0}, x\right)\right|^{2}\left(1+|x|^{2}\right)^{m / 2} d x+C \frac{C_{2}}{C_{1}} .
\end{gathered}
$$

Hence $U \in L^{\infty}\left([0, \infty), L^{2}\left(\left(1+|x|^{2}\right)^{m / 2}\right)\right)$. Since $f_{0}$ is bounded away from zero, the $L^{2}$ bound on $V$ follows.

Concerning the bound on $\nabla_{x} V$, notice that

$$
V(\tau, x)=\frac{U(\tau, x)}{\tilde{U}(\tau, x)}\left(h_{m}(x)+e^{-\tau} \frac{f_{1}(z)}{f_{0}(z)} \cdot \nabla_{x} h_{m}(x)\right),
$$

and thus

$$
\left|\nabla_{x} V(\tau, x)\right| \leq C h_{m}(x)\left(\left|\nabla_{x}\left(\frac{U(\tau, x)}{\tilde{U}(\tau, x)}\right)\right|+\left|\frac{U(\tau, x)}{\tilde{U}(\tau, x)}\right|\right) .
$$


Consequently, for all $\tau \geq 0$,

$$
\int_{\mathbb{R}^{N}}\left|\nabla_{x} V(\tau, x)\right|^{2}\left(1+|x|^{2}\right)^{m / 2} d x \leq C\left\|\frac{U(\tau)}{\tilde{U}(\tau)}\right\|_{H^{1}\left(h_{m}\right)}^{2},
$$

which leads to the bound on $\nabla_{x} V$. Notice that in fact we recover

$$
\nabla_{x} V \in L_{\text {loc }}^{2}\left([0, \infty), L^{2}\left(\left(1+|x|^{2}\right)^{m / 2}\right)\right) .
$$

Hence Proposition 3.2 is proved.

Let us now conclude this section by explaining how the bound (1.2) on the moments of order four follows from (3.2). Let $U_{\text {ini }} \in L^{2}\left(h_{m}^{-1}\right)$, with $m>2(N+2)$ sufficiently large. Then we have proved that $U \in L^{\infty}\left([0, \infty), L^{2}\left(h_{m}^{-1}\right)\right)$, with no restriction on the size of $\left\|U_{\text {ini }}\right\|_{L^{1}\left(\mathbb{R}^{N}\right)}$ in the linear case. Now, for all $\tau \geq 0$, using a simple Hölder inequality, we infer that

$$
\int_{\mathbb{R}^{N}}|U(\tau, x)||x|^{4} d x \leq\|U(\tau)\|_{L^{2}\left(h_{m}^{-1}\right)}\left(\int_{\mathbb{R}^{N}}|x|^{8}\left(1+|x|^{2}\right)^{-m / 2} d x\right)^{1 / 2} .
$$

Hence, if $m>N+8$, we deduce that $U \in L^{\infty}\left([0, \infty), L^{1}\left(|x|^{4}\right)\right)$; going back to the original variables, this entails that (1.2) is satisfied. Thus the convergence result (1.3) holds if the flux $A$ is linear, and Proposition 1.1 is proved.

\section{Long time behaviour}

This section is devoted to the rest of the proof of Theorem 1.1. The idea is to use the $L^{1}$ compactness proved in the previous section (see Proposition 3.2) together with techniques from dynamical systems theory. This type of proof was initiated by S. Osher and J. Ralston in [20], in which the authors proved the $L^{1}$ stability of travelling waves for a quasilinear parabolic equation. Their arguments were then adapted successfully to various kinds of problems in the context of scalar conservation laws (see for instance the review in [22]).

In the present study, our scheme of proof is in fact closely related to the one of M. Escobedo and E. Zuazua in [12]; indeed, the idea is to apply the dynamical systems tools to the rescaled parabolic system (2.3) rather than the original conservation law (1.1). The main difference from [12] lies in the presence of highly oscillating coefficients in (2.3); thus it is necessary to work simultaneously with the homogenized equation (2.13)-(2.15) and with the oscillating one.

Let us now introduce some notation and definitions. First, we denote by $S_{\tau}(\tau \geq 0)$ the semigroup associated with the homogenized equation, that is, equation (2.15) if $N=1$, and (2.13) if $N \geq 2$. According to Proposition 2.1, the semigroup $S_{\tau}$ is well-defined in $L^{\infty}\left(\mathbb{R}^{N}\right) \cap L^{2}(K)$, where $K$ is the weight function defined by (2.19)-(2.20); additionally, the $L^{1}$ contraction property holds, namely

$$
\left\|S_{\tau} F_{1}-S_{\tau} F_{2}\right\|_{L^{1}\left(\mathbb{R}^{N}\right)} \leq\left\|F_{1}-F_{2}\right\|_{L^{1}\left(\mathbb{R}^{N}\right)} \quad \forall \tau \geq 0, \forall F_{1}, F_{2} \in L^{\infty}\left(\mathbb{R}^{N}\right) \cap L^{2}(K) .
$$

Hence $S_{\tau}$ can be extended on $L^{1}\left(\mathbb{R}^{N}\right)$. 
We also define the $\omega$-limit set associated with a given function $U_{\text {ini }} \in L^{1}\left(\mathbb{R}^{N}\right)$ : recalling the definition of the function $V$ (see (3.1)), we set

$$
\Omega\left[U_{\mathrm{ini}}\right]:=\left\{\bar{V} \in L^{1}\left(\mathbb{R}^{N}\right): \exists \tau_{n} \rightarrow \infty, V\left(\tau_{n}\right) \rightarrow \bar{V} \text { in } L^{1}\left(\mathbb{R}^{N}\right)\right\},
$$

where the function $U$ in (3.1) is the unique solution of (2.3) with initial data $U_{\text {ini. }}$. When there is no ambiguity, we will simply write $\Omega$ instead of $\Omega\left[U_{\text {ini }}\right]$.

Notice that $V\left(\tau_{n}\right)$ converges to $\bar{V}$ in $L^{1}$ if and only if

$$
\lim _{n \rightarrow \infty} \int_{\mathbb{R}^{N}}\left|U\left(\tau_{n}, x\right)-f_{0}\left(e^{\tau_{n}} x+c \frac{e^{2 \tau_{n}}-1}{2}\right) \bar{V}(x)\right| d x=0 .
$$

This equivalence will be used repeatedly throughout the section.

The organisation of this section is the following: we first introduce a "quasi-Lyapunov function" for the semigroup associated with equation (2.3). We then prove that Proposition 3.1 holds when the initial data $U_{\text {ini }}$ has a sufficiently small $L^{1}$ norm. Eventually, we prove Proposition 3.1 in the general case.

\subsection{A quasi-Lyapunov function}

Let us first recall the definition of a Lyapunov function (see [17]):

Definition 4.1. Let $\mathcal{X}$ be a Banach space, and let $\pi:[0, \infty) \times \mathcal{X} \rightarrow \mathcal{X}$ be a dynamical system on $\mathcal{X}$, i.e. $\pi$ satisfies the following:

(i) $\pi(0, x)=x$ for all $x \in \mathcal{X}$;

(ii) Semigroup property: For all $s, t \geq 0$ and $x \in \mathcal{X}, \pi(t+s, x)=\pi(t, \pi(s, x))$;

(iii) $\pi$ is continuous on $[0, \infty) \times \mathcal{X}$.

A function $\mathcal{V}: \mathcal{X} \rightarrow \mathbb{R}$ is said to be a Lyapunov function if

(i) $\mathcal{V}$ is continuous;

(ii) $\dot{\mathcal{V}}(x) \leq 0$ for all $x \in \mathcal{X}$, where

$$
\dot{\mathcal{V}}(x):=\liminf _{t \rightarrow 0^{+}} \frac{\mathcal{V}(\pi(t, x))-\mathcal{V}(x)}{t} .
$$

Lyapunov functions are crucial in the analysis of the stability of dynamical systems. In the present context, because of the different scales involved, it seems difficult to find a suitable Lyapunov function, and we thus extend the previous definition:

Definition 4.2. Let $\mathcal{X}$ be a Banach space, and let $t \in[0, \infty) \mapsto x_{t} \in \mathcal{X}$ be a continuous curve in $\mathcal{X}$. Let $\mathcal{H}:[0, \infty) \times \mathcal{X} \rightarrow \mathbb{R}$ be continuous. We say that $\mathcal{H}$ is a quasi-Lyapunov function for the trajectory $\left(x_{t}\right)_{t \geq 0}$ if the following properties hold:

(i) The family $\mathcal{H}\left(t, x_{t}\right)(t \geq 0)$ is bounded in $\mathbb{R}$;

(ii) There exists a function $\psi:[0, \infty) \rightarrow[0, \infty)$ such that $\lim _{t \rightarrow \infty} \psi(t)=0$ and

$$
\forall t \geq 0, \quad \sup _{s \geq t}\left(\mathcal{H}\left(s, x_{s}\right)-\mathcal{H}\left(t, x_{t}\right)\right) \leq \psi(t) .
$$


In other words, we drop the semigroup property associated with the dynamical system $\pi$, and we focus on a particular trajectory; moreover, inequality (4.2) means that $\mathcal{H}\left(s, x_{s}\right)$ is "almost decreasing" with respect to $s$ for large $s$. Notice that a Lyapunov function associated with a semigroup $\pi$ is decreasing along any trajectory $\pi(s, x)(s \geq 0)$.

We then have the following result:

Lemma 4.1. Let $\mathcal{X}$ be a Banach space, and let $t \in[0, \infty) \mapsto x_{t} \in \mathcal{X}$ be a continuous curve in $\mathcal{X}$. Let $\mathcal{H}:[0, \infty) \times \mathcal{X} \rightarrow \mathbb{R}$ be a quasi-Lyapunov function for $\left(x_{t}\right)_{t \geq 0}$. Then $\mathcal{H}\left(t, x_{t}\right)$ has a finite limit as $t \rightarrow \infty$.

Proof. First, since $\mathcal{H}\left(t, x_{t}\right)$ is bounded for $t \in[0, \infty)$, the quantities

$$
\underline{H}:=\liminf _{t \rightarrow \infty} \mathcal{H}\left(t, x_{t}\right), \quad \bar{H}:=\limsup _{t \rightarrow \infty} \mathcal{H}\left(t, x_{t}\right)
$$

are well-defined and belong to $\mathbb{R}$, with $\underline{H} \leq \bar{H}$.

Let $\varepsilon>0$ be arbitrary. There exists $t_{\varepsilon}>0$ such that

$$
\psi(t) \leq \varepsilon \quad \forall t \geq t_{\varepsilon}
$$

By the definition of $\underline{H}$, there exists $s_{\varepsilon} \geq t_{\varepsilon}$ such that

$$
\left|\mathcal{H}\left(s_{\varepsilon}, x_{s_{\varepsilon}}\right)-\underline{H}\right| \leq \varepsilon .
$$

Since $\mathcal{H}$ is a quasi-Lyapunov function, for all $s \geq s_{\varepsilon}$ we have

$$
\mathcal{H}\left(s, x_{s}\right) \leq \mathcal{H}\left(s_{\varepsilon}, x_{s_{\varepsilon}}\right)+\psi\left(s_{\varepsilon}\right) \leq \underline{H}+2 \varepsilon .
$$

Hence

$$
\bar{H} \leq \underline{H}+2 \varepsilon \quad \forall \varepsilon>0,
$$

and $\bar{H}=\underline{H}$. Thus the quantity $\mathcal{H}\left(t, x_{t}\right)$ has a finite limit as $t \rightarrow \infty$.

We now apply this notion to the present context:

Lemma 4.2. Let $M \in \mathbb{R}$ be arbitrary, and let $U_{\mathrm{ini}} \in L^{1}\left(\mathbb{R}^{N}\right)$. For $\tau \geq 0$ and $u \in$ $L^{1}\left(\mathbb{R}^{N}\right)$, define the function $\mathcal{H}$ by

$$
\mathcal{H}(\tau, u):=\int_{\mathbb{R}^{N}}\left|u(x)-U^{\mathrm{app}}\left[F_{M}\right]\left(\tau, x ; e^{\tau}\right)\right| d x,
$$

where the function $U^{\mathrm{app}}$ was introduced in Definition 2.1. Let $\left.U \in \mathcal{C}(\mid 0, \infty), L^{1}\left(\mathbb{R}^{N}\right)\right)$ be the solution of (2.3) with initial data $U_{\tau=0}=U_{\text {ini. }}$ Then $\mathcal{H}$ is a quasi-Lyapunov function for the trajectory $\{U(\tau))\}_{\tau \geq 0}$ in $L^{1}\left(\mathbb{R}^{N}\right)$. As a consequence, the function

$$
\tau \in[0, \infty) \mapsto \int_{\mathbb{R}^{N}}\left|U(\tau, x)-f_{0}(z) F_{M}(x)\right| d x \quad \text { with } \quad z=e^{\tau} x+c \frac{e^{2 \tau}-1}{2}
$$

converges as $\tau \rightarrow \infty$. 
Proof. This property is an easy consequence of Lemma 2.5(i); indeed, according to that lemma, there exists a constant $C$, depending only on $N$ and $M$, such that

$$
\frac{d}{d \tau} \mathcal{H}(\tau, U(\tau))=\frac{d}{d \tau}\left\|U(\tau)-U^{\mathrm{app}}\left[F_{M}\right](\tau)\right\|_{L^{1}\left(\mathbb{R}^{N}\right)} \leq C e^{-\tau} .
$$

Consequently, for all $\tau^{\prime} \geq \tau \geq 0$, we have

$$
\mathcal{H}\left(\tau^{\prime}, U\left(\tau^{\prime}\right)\right)-\mathcal{H}(\tau, U(\tau)) \leq C\left(e^{-\tau}-e^{-\tau^{\prime}}\right) \leq C e^{-\tau}
$$

Thus property (ii) of Definition 4.2 is satisfied. Additionally, notice that

$$
\begin{aligned}
0 \leq \mathcal{H}(\tau, U(\tau)) \leq & \|U(\tau)\|_{L^{1}\left(\mathbb{R}^{N}\right)}+\left\|f_{0}\right\|_{L^{\infty}\left(\mathbb{T}^{N}\right)}\left\|F_{M}\right\|_{L^{1}\left(\mathbb{R}^{N}\right)} \\
& +C e^{-\tau}\left(\left\|\nabla F_{M}\right\|_{L^{1}\left(\mathbb{R}^{N}\right)}+\left\|F_{M}\right\|_{L^{2}\left(\mathbb{R}^{N}\right)}^{2}\right) \\
& +C e^{-2 \tau}\left(\left\|F_{M}\right\|_{W^{2,1}\left(\mathbb{R}^{N}\right)}+\left\|F_{M}\right\|_{L^{3}\left(\mathbb{R}^{N}\right)}^{3}+\left\|\nabla F_{M}\right\|_{L^{2}\left(\mathbb{R}^{N}\right)}^{2}\right) \\
\leq & \left\|U_{\text {ini }}\right\|_{L^{1}\left(\mathbb{R}^{N}\right)}+C .
\end{aligned}
$$

Hence $\mathcal{H}(\tau, U(\tau))$ is bounded for $\tau \in[0, \infty)$. Additionally the continuity of $\mathcal{H}$ on $[0, \infty) \times L^{1}$ is obvious. Consequently, $\mathcal{H}$ is a quasi-Lyapunov function for the trajectory $U(\tau)$. According to Lemma 4.1, $\mathcal{H}(\tau, U(\tau))$ has a finite limit as $\tau \rightarrow \infty$. Furthermore, we have

$$
\left\|U(\tau)-f_{0}(z) F_{M}\right\|_{L^{1}\left(\mathbb{R}^{N}\right)}=\left\|U(\tau)-U^{\mathrm{app}}\left[F_{M}\right](\tau)+e^{-\tau} U_{1}+e^{-2 \tau} U_{2}\right\|_{L^{1}\left(\mathbb{R}^{N}\right)},
$$

where $U_{1}$ and $U_{2}$ are defined by (2.8)-(2.11) and (2.12) respectively. Hence for all $\tau \geq 0$,

$$
\mathcal{H}(\tau, U(\tau))-C e^{-\tau} \leq\left\|U(\tau)-f_{0}(z) F_{M}\right\|_{L^{1}\left(\mathbb{R}^{N}\right)} \leq \mathcal{H}(\tau, U(\tau))+C e^{-\tau},
$$

where the constant $C$ depends only on $W^{s, p}$ bounds on $F_{M}$. Thus the function

$$
\tau \mapsto\left\|U(\tau)-f_{0}(z) F_{M}\right\|_{L^{1}\left(\mathbb{R}^{N}\right)}
$$

converges as $\tau \rightarrow \infty$, and

$$
\lim _{\tau \rightarrow \infty}\left\|U(\tau)-f_{0}(z) F_{M}\right\|_{L^{1}\left(\mathbb{R}^{N}\right)}=\lim _{\tau \rightarrow \infty} \mathcal{H}(\tau, U(\tau)) .
$$

Definition 4.3. Let $U_{\text {ini }} \in L^{1}\left(\mathbb{R}^{N}\right)$ be arbitrary, and let $M:=\int_{\mathbb{R}^{N}} U_{\text {ini. }}$. Let $U$ be the solution of (2.3) with initial data $U_{\mid t=0}=U_{\text {ini }}$. We define the number $\ell\left(U_{\text {ini }}\right)$ by

$$
\ell\left(U_{\text {ini }}\right):=\lim _{\tau \rightarrow \infty} \int_{\mathbb{R}^{N}}\left|U(\tau, x)-f_{0}(z) F_{M}(x)\right| d x \quad \text { with } \quad z=e^{\tau} x+c \frac{e^{2 \tau}-1}{2} .
$$

Notice that Proposition 3.1 is equivalent to

$$
\ell\left(U_{\text {ini }}\right)=0 \quad \forall U_{\text {ini }} \in L^{1}\left(\mathbb{R}^{N}\right) .
$$

Classically, we now derive a continuity property for the function $\ell$ :

Lemma 4.3. The function $U \in L^{1}\left(\mathbb{R}^{N}\right) \mapsto \ell(U) \in \mathbb{R}$ is Lipschitz continuous. 
Proof. Let $U_{\mathrm{ini}}^{(1)}, U_{\mathrm{ini}}^{(2)} \in L^{1}\left(\mathbb{R}^{N}\right)$, and let $M^{(i)}=\int_{\mathbb{R}^{N}} U_{\mathrm{ini}}^{(i)}$ for $i=1,2$. We denote by $U^{(i)} \in \mathcal{C}\left([0, \infty), L^{1}\left(\mathbb{R}^{N}\right)\right)$ the solution of (2.3) with initial data $U_{\text {ini }}^{(i)}$. Then for all $\tau \geq 0$, the $L^{1}$ contraction principle ensures that

$$
\left\|U^{(1)}(\tau)-U^{(2)}(\tau)\right\|_{L^{1}\left(\mathbb{R}^{N}\right)} \leq\left\|U_{\mathrm{ini}}^{(1)}-U_{\mathrm{ini}}^{(2)}\right\|_{L^{1}\left(\mathbb{R}^{N}\right)} .
$$

Hence, for all $\tau \geq 0$, we have

$$
\begin{aligned}
\left|\int_{\mathbb{R}^{N}}\right| U^{(1)}(\tau, x)- & f_{0}(z) F_{M^{(1)}}(x)\left|d x-\int_{\mathbb{R}^{N}}\right| U^{(2)}(\tau, x)-f_{0}(z) F_{M^{(2)}}(x)|d x| \\
& \leq\left\|U^{(1)}(\tau)-U^{(2)}(\tau)\right\|_{L^{1}\left(\mathbb{R}^{N}\right)}+\left\|f_{0}\right\|_{L^{\infty}\left(\mathbb{T}^{N}\right)}\left\|F_{M^{(1)}}-F_{M^{(2)}}\right\|_{L^{1}\left(\mathbb{R}^{N}\right)} .
\end{aligned}
$$

According to Lemma A.2 in the Appendix,

$$
\left\|F_{M^{(1)}}-F_{M^{(2)}}\right\|_{L^{1}\left(\mathbb{R}^{N}\right)}=\left|M^{(1)}-M^{(2)}\right| \leq\left\|U_{\mathrm{ini}}^{(1)}-U_{\mathrm{ini}}^{(2)}\right\|_{L^{1}\left(\mathbb{R}^{N}\right)} .
$$

Eventually, we obtain, for all $\tau \geq 0$,

$$
\begin{aligned}
\left|\int_{\mathbb{R}^{N}}\right| U^{(1)}(\tau, x)-f_{0}(z) F_{M^{(1)}}(x) \mid d x-\int_{\mathbb{R}^{N}} & \left|U^{(2)}(\tau, x)-f_{0}(z) F_{M^{(2)}}(x)\right| d x \mid \\
& \leq\left(1+\left\|f_{0}\right\|_{L^{\infty}\left(\mathbb{T}^{N}\right)}\right)\left\|U_{\mathrm{ini}}^{(1)}-U_{\mathrm{ini}}^{(2)}\right\|_{L^{1}\left(\mathbb{R}^{N}\right)},
\end{aligned}
$$

and thus, passing to the limit,

$$
\left|\ell\left(U_{\mathrm{ini}}^{(1)}\right)-\ell\left(U_{\mathrm{ini}}^{(2)}\right)\right| \leq\left(1+\left\|f_{0}\right\|_{L^{\infty}\left(\mathbb{T}^{N}\right)}\right)\left\|U_{\mathrm{ini}}^{(1)}-U_{\mathrm{ini}}^{(2)}\right\|_{L^{1}\left(\mathbb{R}^{N}\right)} .
$$

Hence $\ell$ is a Lipschitz continuous function.

\subsection{Analysis of the $\omega$-limit set}

Proposition 4.1. Let $U_{\mathrm{ini}} \in L^{1}\left(\mathbb{R}^{N}\right)$, and set $M:=\int_{\mathbb{R}^{N}} U_{\mathrm{ini}}$. Assume that the $\omega$-limit set $\Omega$ associated with $U_{\mathrm{ini}}$ is nonempty (see (4.1)). Then the following properties hold:

(i) for all $\bar{V} \in \Omega, \int_{\mathbb{R}^{N}} \bar{V}=M$;

(ii) $S_{\tau} \Omega \subset \Omega$ for all $\tau \geq 0$;

(iii) for all $\bar{V} \in \Omega$, we have $\left\|\bar{V}-F_{M}\right\|_{L^{1}\left(\mathbb{R}^{N}\right)}=\ell\left(U_{\text {ini }}\right)$.

Proof. Throughout the proof, we denote by $U$ the unique solution of equation (2.3) with initial data $U_{\text {ini }}$.

Property (i) is quite straightforward: indeed, conservation of mass for the equation (2.3) implies that

$$
\int_{\mathbb{R}^{N}} U(\tau)=M \quad \forall \tau \geq 0
$$

If $\bar{V} \in \Omega$, then there exists a sequence $\left(\tau_{n}\right)_{n \geq 0}$ such that

$$
\lim _{n \rightarrow \infty} \tau_{n}=\infty \quad \text { and } \quad \lim _{n \rightarrow \infty} \int_{\mathbb{R}^{N}}\left|U\left(\tau_{n}, x\right)-f_{0}\left(z_{n}\right) \bar{V}(x)\right| d x=0,
$$


where $z_{n}=e^{\tau_{n}} x+c\left(e^{2 \tau_{n}}-1\right) / 2$. According to a result of G. Allaire (see [2]),

$$
\lim _{n \rightarrow \infty} \int_{\mathbb{R}^{N}} f_{0}\left(z_{n}\right) \bar{V}(x) d x=\left\langle f_{0}\right\rangle \int_{\mathbb{R}^{N}} \bar{V}=\int_{\mathbb{R}^{N}} \bar{V}
$$

gathering the three equalities, we obtain property (i).

We now address the proof of property (ii), which relies on Lemma 2.5(ii); let $\bar{V} \in \Omega$ be arbitrary, and for all $\varepsilon>0$, let $\bar{V}_{\varepsilon} \in L^{2}(K) \cap L^{\infty}\left(\mathbb{R}^{N}\right)$ be such that

$$
\left\|\bar{V}_{\varepsilon}-\bar{V}\right\|_{L^{1}\left(\mathbb{R}^{N}\right)} \leq \varepsilon
$$

Let $\left(\tau_{n}\right)_{n \geq 0}$ be a sequence of positive numbers such that $\tau_{n} \rightarrow \infty$ and

$$
\int_{\mathbb{R}^{N}}\left|U\left(\tau_{n}, x\right)-f_{0}\left(z_{n}\right) \bar{V}(x)\right| d x \rightarrow 0,
$$

where $z_{n}=e^{\tau_{n}} x+c\left(e^{2 \tau_{n}}-1\right) / 2$.

Let $\rho \in \mathcal{C}_{0}^{\infty}\left(\mathbb{R}^{N}\right)$ be a mollifying kernel; for $\delta>0$, set $\rho_{\delta}:=\delta^{-N} \rho(\cdot / \delta)$, and define the function $U_{n}^{\delta, \varepsilon}$ by

$$
U_{n}^{\delta, \varepsilon}(\tau, x):=U^{\mathrm{app}}\left[\left(S_{\tau} \bar{V}_{\varepsilon}\right) *_{x} \rho_{\delta}\right]\left(\tau, x ; e^{\tau_{n}+\tau}\right) .
$$

Then Lemma 2.5 ensures that $U_{n}^{\delta, \varepsilon}$ satisfies equation (2.3) with an error term, bounded for all $T>0$ in $L^{\infty}\left([0, T], L^{1}\left(\mathbb{R}^{N}\right)\right)$ by

$$
\omega_{T, \varepsilon}(\delta)+C_{T, \varepsilon, \delta} e^{-\tau_{n}}
$$

where $\omega_{T, \varepsilon}:[0, \infty) \rightarrow[0, \infty)$ is such that $\lim _{0^{+}} \omega_{T, \varepsilon}=0$, and the constant $C_{T, \varepsilon, \delta}$ depends only on $\varepsilon, \delta, N$ and $T$.

Using the $L^{1}$ contraction principle for scalar conservation laws, we infer that for all $T>0$, and all $\tau \in[0, T]$,

$$
\begin{aligned}
\int_{\mathbb{R}^{N}} \mid U\left(\tau_{n}+\tau, x\right)- & U_{n}^{\delta, \varepsilon}(\tau, x) \mid d x \\
\leq & \omega_{T, \varepsilon}(\delta)+C_{T, \varepsilon, \delta} e^{-\tau_{n}}+\int_{\mathbb{R}^{N}}\left|U\left(\tau_{n}, x\right)-U_{n \mid \tau=0}^{\delta, \varepsilon}(x)\right| d x \\
\leq & \omega_{T, \varepsilon}(\delta)+C_{T, \varepsilon, \delta} e^{-\tau_{n}}+\int_{\mathbb{R}^{N}}\left|U\left(\tau_{n}, x\right)-f_{0}\left(z_{n}\right) \bar{V}(x)\right| d x \\
& +\int_{\mathbb{R}^{N}}\left|U_{n \mid \tau=0}^{\delta, \varepsilon}(x)-f_{0}\left(z_{n}\right) \bar{V}(x)\right| d x .
\end{aligned}
$$

Now, according to Definition 2.1,

$$
\begin{aligned}
U_{n}^{\delta, \varepsilon}(\tau, x)= & \left(S_{\tau} \bar{V}_{\varepsilon}\right) *_{x} \rho_{\delta}(x) f_{0}\left(e^{\tau_{n}+\tau} x+c \frac{e^{2\left(\tau_{n}+\tau\right)}-1}{2}\right) \\
& +e^{-\left(\tau_{n}+\tau\right)} U_{1}^{\delta, \varepsilon}\left(\tau, x, e^{\tau_{n}+\tau} x+c \frac{e^{2\left(\tau_{n}+\tau\right)}-1}{2}\right) \\
& +e^{-2\left(\tau_{n}+\tau\right)} V_{2}^{\delta, \varepsilon}\left(\tau, x, e^{\tau_{n}+\tau} x+c \frac{e^{2\left(\tau_{n}+\tau\right)}-1}{2}\right) .
\end{aligned}
$$


Hence for all $\tau \in[0, T]$, we have

$$
\begin{aligned}
\int_{\mathbb{R}^{N}} \mid U_{n}^{\delta, \varepsilon}(\tau, x) & -S_{\tau} \bar{V} f_{0}\left(e^{\tau_{n}+\tau} x+c \frac{e^{2\left(\tau_{n}+\tau\right)}-1}{2}\right) \mid d x \\
\leq & \left\|f_{0}\right\|_{\infty} \sup _{\tau \in[0, T]}\left\|S_{\tau} \bar{V}-\left(S_{\tau} \bar{V}_{\varepsilon}\right) * x \rho_{\delta}\right\|_{L^{1}\left(\mathbb{R}^{N}\right)} \\
& +e^{-\tau_{n}}\left(\left\|U_{1}^{\delta, \varepsilon}\right\|_{L^{\infty}\left([0, T] \times \mathbb{T}_{z}^{N}, L^{1}\left(\mathbb{R}_{x}^{N}\right)\right)}+\left\|V_{2}^{\delta, \varepsilon}\right\|_{L^{\infty}\left([0, T] \times \mathbb{T}_{z}^{N}, L^{1}\left(\mathbb{R}_{x}^{N}\right)\right)}\right) \\
\leq & \left\|f_{0}\right\|_{\infty}\left[\left\|\bar{V}-\bar{V}_{\varepsilon}\right\|_{L^{1}}+\sup _{\tau \in[0, T]}\left\|S_{\tau} \bar{V}_{\varepsilon}-\left(S_{\tau} \bar{V}_{\varepsilon}\right) *_{x} \rho_{\delta}\right\|_{L^{1}\left(\mathbb{R}^{N}\right)}\right] \\
& +e^{-\tau_{n}}\left(\left\|U_{1}^{\delta, \varepsilon}\right\|_{L^{\infty}\left([0, T] \times \mathbb{T}_{z}^{N}, L^{1}\left(\mathbb{R}_{x}^{N}\right)\right)}+\left\|V_{2}^{\delta, \varepsilon}\right\|_{L^{\infty}\left([0, T] \times \mathbb{T}_{z}^{N}, L^{1}\left(\mathbb{R}_{x}^{N}\right)\right)}\right) \\
\leq & C \varepsilon+\omega_{T, \varepsilon}(\delta)+C_{T, \varepsilon, \delta} e^{-\tau_{n}} .
\end{aligned}
$$

Gathering the two inequalities, we deduce that for all $n, \delta, \varepsilon$,

$$
\begin{aligned}
\sup _{\tau \in[0, T]} \int_{\mathbb{R}^{N}}\left|U\left(\tau_{n}+\tau, x\right)-S_{\tau} \bar{V}(x) f_{0}\left(e^{\tau_{n}+\tau} x+c \frac{e^{2\left(\tau_{n}+\tau\right)}-1}{2}\right)\right| d x \\
\leq C \varepsilon+\omega_{T, \varepsilon}(\delta)+C_{T, \varepsilon, \delta} e^{-\tau_{n}}+\int_{\mathbb{R}^{N}}\left|U\left(\tau_{n}, x\right)-f_{0}\left(z_{n}\right) \bar{V}(x)\right| d x
\end{aligned}
$$

In the right-hand side of the above inequality, we first choose $\varepsilon$ sufficiently small, then $\delta$ so that $\omega_{T, \varepsilon}(\delta)$ is sufficiently small, and eventually $n$ large enough so that the remaining two terms are small as well; hence

$$
\lim _{n \rightarrow \infty} \inf _{\varepsilon, \delta>0}\left(C \varepsilon+\omega_{T, \varepsilon}(\delta)+C_{T, \varepsilon, \delta} e^{-\tau_{n}}+\int_{\mathbb{R}^{N}}\left|U\left(\tau_{n}, x\right)-f_{0}\left(z_{n}\right) \bar{V}(x)\right| d x\right)=0 .
$$

Thus we have proved that for all $T>0$,

$$
\lim _{n \rightarrow \infty} \sup _{\tau \in[0, T]} \int_{\mathbb{R}^{N}}\left|U\left(\tau_{n}+\tau, x\right)-\left(S_{\tau} \bar{V}\right) f_{0}\left(e^{\tau_{n}+\tau} x+c \frac{e^{2\left(\tau_{n}+\tau\right)}-1}{2}\right)\right| d x=0 .
$$

The above convergence entails immediately that $S_{\tau} \bar{V} \in \Omega$ for all $\tau \in[0, T]$. Since $T>0$ was arbitrary, property (ii) is proved.

It remains to prove property (iii), which is a variant of the LaSalle invariance principle; let $\bar{V} \in \Omega$ be arbitrary, and let $\tau_{n}$ be a sequence of positive numbers such that $\lim _{n \rightarrow \infty} \tau_{n}=+\infty$ and

$$
\lim _{n \rightarrow \infty} \int_{\mathbb{R}^{N}}\left|U\left(\tau_{n}, x\right)-f_{0}\left(z_{n}\right) \bar{V}(x)\right| d x=0,
$$

where $z_{n}=e^{\tau_{n}} x+c\left(e^{2 \tau_{n}}-1\right) / 2$. According to a result of G. Allaire (see [2]), we have, 
since $\left\langle f_{0}\right\rangle=1$ and $f_{0} \in \mathcal{C}\left(\mathbb{T}^{N}\right)$,

$$
\begin{aligned}
\left\|\bar{V}-F_{M}\right\|_{L^{1}\left(\mathbb{R}^{N}\right)} & =\lim _{n \rightarrow \infty} \int_{\mathbb{R}^{N}} f_{0}\left(z_{n}\right)\left|\bar{V}(x)-F_{M}(x)\right| d x \\
& =\lim _{n \rightarrow \infty} \int_{\mathbb{R}^{N}} f_{0}\left(z_{n}\right)\left|\bar{V}(x)-\frac{U\left(\tau_{n}, x\right)}{f_{0}\left(z_{n}\right)}+\frac{U\left(\tau_{n}, x\right)}{f_{0}\left(z_{n}\right)}-F_{M}(x)\right| d x \\
& =\lim _{n \rightarrow \infty} \int_{\mathbb{R}^{N}} f_{0}\left(z_{n}\right)\left|\frac{U\left(\tau_{n}, x\right)}{f_{0}\left(z_{n}\right)}-F_{M}(x)\right| d x=\ell\left(U_{\mathrm{ini}}\right) .
\end{aligned}
$$

Consequently, $\left\|\bar{V}-F_{M}\right\|_{L^{1}\left(\mathbb{R}^{N}\right)}=\ell\left(U_{\text {ini }}\right)$ for all $\bar{V} \in \Omega$.

Corollary 4.1. Let $U_{\mathrm{ini}} \in L^{1}\left(\mathbb{R}^{N}\right)$, and set $M:=\int_{\mathbb{R}^{N}} U_{\mathrm{ini}}$. Assume that the $\omega$-limit set $\Omega\left[U_{\mathrm{ini}}\right]$ is nonempty. Then $\ell\left(U_{\mathrm{ini}}\right)=0$, and thus the conclusion of Proposition 3.1 holds.

Proof. Let $\bar{V} \in \Omega$ be arbitrary. Then

$$
\lim _{\tau \rightarrow \infty}\left\|S_{\tau} \bar{V}-F_{M}\right\|_{L^{1}\left(\mathbb{R}^{N}\right)}=0 ;
$$

this property is stated in Proposition 2.1 in the case when $\bar{V} \in L^{\infty}\left(\mathbb{R}^{N}\right) \cap L^{2}(K)$, but can be in fact easily generalized to an arbitrary function $\bar{V} \in L^{1}$ by using the contractivity of the semigroup $S_{\tau}$ : indeed, let $\varepsilon>0$, and let $\bar{V}_{\varepsilon} \in L^{\infty}\left(\mathbb{R}^{N}\right) \cap L^{2}(K)$ be such that $\int \bar{V}_{\varepsilon}=\int \bar{V}=M$, and

$$
\left\|\bar{V}_{\varepsilon}-\bar{V}\right\|_{L^{1}\left(\mathbb{R}^{N}\right)} \leq \varepsilon
$$

Then for all $\tau \geq 0$,

$$
\begin{aligned}
\left\|S_{\tau} \bar{V}-F_{M}\right\|_{L^{1}\left(\mathbb{R}^{N}\right)} & \leq\left\|S_{\tau} \bar{V}-S_{\tau} \bar{V}_{\varepsilon}\right\|_{L^{1}\left(\mathbb{R}^{N}\right)}+\left\|S_{\tau} \bar{V}_{\varepsilon}-F_{M}\right\|_{L^{1}\left(\mathbb{R}^{N}\right)} \\
& \leq\left\|\bar{V}-\bar{V}_{\varepsilon}\right\|_{L^{1}\left(\mathbb{R}^{N}\right)}+\left\|S_{\tau} \bar{V}_{\varepsilon}-F_{M}\right\|_{L^{1}\left(\mathbb{R}^{N}\right)}
\end{aligned}
$$

Hence, using Proposition 2.1, we infer that

$$
\limsup _{\tau \rightarrow \infty}\left\|S_{\tau} \bar{V}-F_{M}\right\|_{L^{1}\left(\mathbb{R}^{N}\right)} \leq \varepsilon \quad \forall \varepsilon>0,
$$

and thus $\left\|S_{\tau} \bar{V}-F_{M}\right\|_{L^{1}\left(\mathbb{R}^{N}\right)}$ vanishes as $\tau \rightarrow \infty$.

On the other hand, Proposition 4.1(i) ensures that $S_{\tau} \bar{V} \in \Omega$ for all $\tau \geq 0$, and thus, using Proposition 4.1(iii),

$$
\left\|S_{\tau} \bar{V}-F_{M}\right\|_{L^{1}\left(\mathbb{R}^{N}\right)}=\ell\left(U_{\text {ini }}\right) \quad \forall \tau \geq 0 .
$$

Consequently, $\ell\left(U_{\text {ini }}\right)=0$. Going back to the definition of $\ell\left(U_{\text {ini }}\right)$, we deduce that

$$
\lim _{\tau \rightarrow \infty} \int_{\mathbb{R}^{N}}\left|U(\tau, x)-f_{0}\left(e^{\tau} x+c \frac{e^{2 \tau}-1}{2}\right) F_{M}(x)\right| d x=0 .
$$

Thus the proof of Proposition 3.1 is complete provided we are able to show that the set $\Omega\left[U_{\mathrm{ini}}\right]$ is nonempty for a sufficiently large class of functions $U_{\mathrm{ini}} \in L^{1}\left(\mathbb{R}^{N}\right)$. In the case when $\left\|U_{\text {ini }}\right\|_{1}$ is small, this result follows from Proposition 3.2 and from a contraction principle. The proof in the general case is more involved, and in fact, an analysis similar to the one performed in Section 3 has to be conducted once more. 
4.3. Proof of Proposition 3.1 when $\left\|U_{\mathrm{ini}}\right\|_{L^{1}\left(\mathbb{R}^{N}\right)}$ is small

We now complete the proof of Theorem 1.1 when $\left\|U_{\text {ini }}\right\|_{L^{1}\left(\mathbb{R}^{N}\right)}$ is small. Let $U_{\text {ini }} \in$ $L^{1}\left(\mathbb{R}^{N}\right)$. Assume that $U_{\text {ini }}$ satisfies the following assumptions:

$$
\begin{array}{ll}
\exists m>2(N+1), \quad & U_{\text {ini }} \in L^{2}\left(\left(1+|x|^{2}\right)^{m / 2}\right) \cap L^{\infty}\left(\mathbb{R}^{N}\right), \\
& \left\|U_{\text {ini }}\right\|_{L^{1}\left(\mathbb{R}^{N}\right)} \leq C_{m},
\end{array}
$$

where the constant $C_{m}$ was introduced in Proposition 3.2. Then according to Proposition 3.2 , the $\omega$-limit set $\Omega\left[U_{\text {ini }}\right]$ is nonempty, and consequently Proposition 3.1 is true (see Corollary 4.1).

Let us now prove that Proposition 3.1 holds when $U_{\text {ini }}$ merely satisfies (4.4): this fact is a direct consequence of the density of $L^{2}\left(\left(1+|x|^{2}\right)^{m / 2}\right) \cap L^{\infty}\left(\mathbb{R}^{N}\right)$ in $L^{1}\left(\mathbb{R}^{N}\right)$, together with the continuity of $\ell$. Indeed, for all $\varepsilon>0$, let $U_{\text {ini }}^{\varepsilon} \in L^{2}\left(\left(1+|x|^{2}\right)^{m / 2}\right) \cap L^{\infty}\left(\mathbb{R}^{N}\right)$ be such that

$$
\left\|U_{\text {ini }}-U_{\text {ini }}^{\varepsilon}\right\|_{L^{1}\left(\mathbb{R}^{N}\right)} \leq \varepsilon, \quad\left\|U_{\text {ini }}^{\varepsilon}\right\|_{L^{1}\left(\mathbb{R}^{N}\right)} \leq C_{m} .
$$

Then $\ell\left(U_{\text {ini }}^{\varepsilon}\right)=0$. Since $\ell$ is Lipschitz continuous (see Lemma 4.3), there exists a constant $C$ such that

$$
\ell\left(U_{\text {ini }}\right)=\left|\ell\left(U_{\text {ini }}\right)-\ell\left(U_{\text {ini }}^{\varepsilon}\right)\right| \leq C\left\|U_{\text {ini }}-U_{\text {ini }}^{\varepsilon}\right\|_{L^{1}\left(\mathbb{R}^{N}\right)} \leq C \varepsilon .
$$

Since the above inequality holds for all $\varepsilon>0$, we deduce that $\ell\left(U_{\text {ini }}\right)=0$. Recalling the definition of $\ell$, we infer that Proposition 3.1 holds for all initial data $U_{\text {ini }} \in L^{1}\left(\mathbb{R}^{N}\right)$ satisfying (4.4).

\subsection{Proof of Proposition 3.1 in the general case}

The case when $\left\|U_{\text {ini }}\right\|_{1}$ is large follows from the following lemma:

Lemma 4.4. There exists a constant $C_{0}$, depending only on $N$ and on the flux $A$, such that for all $U_{\mathrm{ini}} \in L^{1}\left(\mathbb{R}^{N}\right)$,

$$
\ell\left(U_{\text {ini }}\right) \leq C_{0} \Rightarrow \ell\left(U_{\text {ini }}\right)=0 .
$$

Before proving the above lemma, let us explain why the result of Proposition 3.1 follows. Since $\ell$ is Lipschitz continuous, $\ell\left(L^{1}\left(\mathbb{R}^{N}\right)\right)$ is a connected subset of $[0, \infty)$, i.e. an interval. Moreover, $0 \in \ell\left(L^{1}\left(\mathbb{R}^{N}\right)\right)$ according to the previous section, and Lemma 4.4 entails that

$$
\ell\left(L^{1}\left(\mathbb{R}^{N}\right)\right) \cap\left(0, C_{0}\right]=\emptyset .
$$

Consequently, $\ell\left(L^{1}\left(\mathbb{R}^{N}\right)\right)=\{0\}$.

It remains to prove Lemma 4.4. According to Corollary 4.1 and using by now standard arguments, we only have to prove that there exists a set $\mathcal{A} \subset L^{1}\left(\mathbb{R}^{N}\right)$, which is dense in $L^{1}\left(\mathbb{R}^{N}\right)$, and such that

$$
\exists C>0, \forall U_{\text {ini }} \in L^{1}\left(\mathbb{R}^{N}\right) \cap \mathcal{A}, \quad \ell\left(U_{\text {ini }}\right) \leq C \Rightarrow \Omega\left[U_{\text {ini }}\right] \neq \emptyset .
$$


In the following, we will take $\mathcal{A}=L^{\infty}\left(\mathbb{R}^{N}\right) \cap L^{2}\left(\left(1+|x|^{2}\right)^{m / 2}\right)$ for some $m>0$ sufficiently large.

The scheme of proof of the implication (4.5) is very similar to the one of Proposition 3.2; indeed, we have to prove that if $\ell\left(U_{\text {ini }}\right)$ is small enough, then there exists a sequence $\left(\tau_{n}\right)$ of positive numbers, with $\lim _{n \rightarrow \infty} \tau_{n}=+\infty$, such that $\left(V\left(\tau_{n}, \cdot\right)\right)_{n \rightarrow \infty}$ is a compact sequence in $L^{1}\left(\mathbb{R}^{N}\right)$. Notice that this is obviously equivalent to the compactness of the sequence $V\left(\tau_{n}, \cdot\right)-F_{M}$, whose $L^{1}$ norm is of the order of $\ell\left(U_{\text {ini }}\right)$ as $n \rightarrow \infty$. Thus our strategy is the following: rather than using directly the equation on $U$, we consider the equation on the function $U-U^{\mathrm{app}}\left[F_{M}\right]$. We prove that for an appropriate function $\tilde{U}$, an inequality of the type (3.6) holds, with $U$ replaced by $U-U^{\text {app }}\left[F_{M}\right]$. Then, all the occurrences of $\|U(\tau)\|_{L^{1}\left(\mathbb{R}^{N}\right)}$ in the proof of Proposition 3.2 are replaced by $\left\|\left(U-U^{\text {app }}\left[F_{M}\right]\right)(\tau)\right\|_{L^{1}\left(\mathbb{R}^{N}\right)}$, which converges towards $\ell\left(U_{\text {ini }}\right)$ as $\tau \rightarrow \infty$. Thus the same arguments which led us to compactness in the case when $\left\|U_{\mathrm{ini}}\right\|_{L^{1}\left(\mathbb{R}^{N}\right)}$ is small show that compactness holds, provided $\ell\left(U_{\text {ini }}\right)$ is small enough.

Let us now retrace the main lines of the proof: first, consider a function $U_{\text {ini }} \in L^{1}\left(\mathbb{R}^{N}\right)$ such that $U_{\text {ini }} \in L^{2}\left(\left(1+|x|^{2}\right)^{m / 2}\right)$ for some sufficiently large $m$ (to be chosen later). Set $M=\int_{\mathbb{R}^{N}} U_{\text {ini }}$ and

$$
W(\tau, x)=U(\tau, x)-U^{\mathrm{app}}\left[F_{M}\right]\left(\tau, x ; e^{\tau}\right) .
$$

In the rest of the proof, for the sake of brevity, we will write $U^{\text {app }}(\tau, x)$ as short-hand for $U^{\text {app }}\left[F_{M}\right]\left(\tau, x ; e^{\tau}\right)$. Then the following properties hold:

$$
\begin{aligned}
& W \in L_{\mathrm{loc}}^{\infty}\left([0, \infty), L^{2}\left(\left(1+|x|^{2}\right)^{m / 2}\right)\right) \cap L_{\mathrm{loc}}^{2}\left([0, \infty), H^{1}\left(\left(1+|x|^{2}\right)^{m / 2}\right)\right), \\
& \exists C>0, \forall \tau \geq 0, \quad\|W(\tau, \cdot)\|_{L^{\infty}\left(\mathbb{R}^{N}\right)} \leq C e^{N \tau} \\
& \lim _{\tau \rightarrow \infty}\|W(\tau)\|_{L^{1}\left(\mathbb{R}^{N}\right)}=\ell\left(U_{\mathrm{ini}}\right)
\end{aligned}
$$

Moreover, using Lemma 2.5, we deduce that $W$ satisfies

$$
\begin{aligned}
\partial_{\tau} W= & \operatorname{div}_{x}(x W)+\Delta_{x} W-R \operatorname{div}_{x}\left(\left(\alpha_{1}(z)-c\right) W\right) \\
& -R^{N+1} \operatorname{div}_{x}\left[\tilde{B}_{1}\left(z, U / R^{N}\right)-\tilde{B}_{1}\left(z, U^{\mathrm{app}} / R^{N}\right)\right]+U^{\mathrm{rem}},
\end{aligned}
$$

with $R=e^{\tau}, z=R x+c\left(R^{2}-1\right) / 2$, and we recall that the remainder $U^{\text {rem }}$ satisfies

$$
\left\|U^{\mathrm{rem}}(\tau)\right\|_{L^{\infty}\left(\mathbb{R}^{N}\right)}+\left\|U^{\mathrm{rem}}(\tau)\right\|_{L^{2}\left(e \gamma|x|^{2}\right)} \leq C e^{-\tau}
$$

for some $\gamma>0$. Then, using the bounds on $U, U^{\text {app }}$ together with the regularity assumptions on $\tilde{B}$, it can be easily proved that

$$
\tilde{B}_{1}\left(z, U(\tau, x) / R^{N}\right)-\tilde{B}_{1}\left(z, U^{\mathrm{app}}(\tau, x) / R^{N}\right)=2 \alpha_{2}(z) f_{0}(z) \frac{F_{M}(x) W(\tau, x)}{R^{2 N}}+b(\tau, x),
$$

and the function $b$ is such that there exists $C>0$ such that

$$
\forall(\tau, x) \in \mathbb{R}_{+} \times \mathbb{R}^{N}, \quad|b(\tau, x)| \leq C\left(\left|\frac{W(\tau, x)}{R^{N}}\right|^{2}+\frac{|W(\tau, x)|}{R^{2 N+1}}+\frac{\left|U^{\text {app }}(\tau, x)\right|^{3}}{R^{3 N}}\right) .
$$


We define a function $\tilde{W}$ by

$$
\tilde{W}(\tau, x)=W_{0}(x, z)+e^{-\tau} W_{1}(x, z),
$$

with $W_{0}(x, z)=f_{0}(z) h_{m}(x)$ and

$-\Delta_{z} W_{1}+\operatorname{div}_{z}\left(\alpha_{1} W_{1}\right)=2 \sum_{i=1}^{N} \frac{\partial^{2} W_{0}}{\partial x_{i} \partial z_{i}}-\operatorname{div}_{x}\left(\left(\alpha_{1}-c\right) W_{0}\right)-2 \mathbf{1}_{N=1} \operatorname{div}_{z}\left(\alpha_{2} f_{0} F_{M} W_{0}\right)$

Notice that by the definition of $f_{0}$ and $c$, the compatibility condition is always satisfied, and

$$
W_{1}(x, z)=f_{1}(z) \cdot \nabla_{x} h_{m}(x)+\mathbf{1}_{N=1} w_{1}(z) F_{M}(x) h_{m}(x)
$$

with

$$
-\Delta_{z} w_{1}+\operatorname{div}_{z}\left(\alpha_{1} w_{1}\right)=-2 \operatorname{div}_{z}\left(\alpha_{2} f_{0}^{2}\right) .
$$

Let $\tau_{0}>0$ be such that

$$
\tilde{W}(\tau, x) \geq \frac{1}{2} f_{0}(z) h_{m}(x) \quad \forall \tau \geq \tau_{0}, \forall y \in \mathbb{R}^{N}
$$

For further purposes, we also choose $\tau_{0}$ such that

$$
\|W(\tau, \cdot)\|_{L^{1}\left(\mathbb{R}^{N}\right)} \leq 2 \ell\left(U_{\text {ini }}\right) \quad \forall \tau \geq \tau_{0}
$$

(Notice that if $\ell\left(U_{\text {ini }}\right)=0$ there is nothing to prove.)

Using calculations similar to the ones in the proof of Proposition 3.2 (cf. (3.6)-(3.8)), we infer that for $\tau \geq \tau_{0}$,

$$
\begin{aligned}
& \frac{d}{d \tau} \int_{\mathbb{R}^{N}}\left|\frac{W}{\tilde{W}}\right|^{2} \tilde{W}(\tau)+\frac{m-N}{4} \int_{\mathbb{R}^{N}}\left|\frac{W}{\tilde{W}}\right|^{2} \tilde{W}(\tau)+2 \int_{\mathbb{R}^{N}}\left|\nabla\left(\frac{W}{\tilde{W}}\right)\right|^{2} \tilde{W}(\tau) \\
& \leq C \int_{\mathbb{R}^{N}}\left(\frac{W(\tau, x)}{\tilde{W}(\tau, x)}\right)^{2} \frac{d x}{\left(1+|x|^{2}\right)^{1+m / 2}} \\
&+C e^{(1-N) \tau} \int_{\mathbb{R}^{N}}|W(\tau, x)|^{2}\left|\nabla\left(\frac{W(\tau, x)}{\tilde{W}(\tau, x)}\right)\right| d x \\
&+C e^{-N \tau} \int_{\mathbb{R}^{N}}|W(\tau, x)| \nabla\left(\frac{W(\tau, x)}{\tilde{W}(\tau, x)}\right) \mid d x \\
&+C e^{(-2 N+1) \tau} \int_{\mathbb{R}^{N}}\left|U^{\mathrm{app}}(\tau, x)\right|^{3}\left|\nabla\left(\frac{W(\tau, x)}{\tilde{W}(\tau, x)}\right)\right| d x \\
&+2 \int_{\mathbb{R}^{N}}\left|\frac{W(\tau, x)}{\tilde{W}(\tau, x)}\right|\left|U^{\mathrm{rem}}(\tau, x)\right| d x .
\end{aligned}
$$


Using the same arguments as in the third step of the proof of Proposition 3.2, we deduce that if $m>2(N+1)$,

$$
\begin{aligned}
C \int_{\mathbb{R}^{N}}\left(\frac{W(\tau, x)}{\tilde{W}(\tau, x)}\right)^{2} \frac{d x}{\left(1+|x|^{2}\right)^{1+m / 2}} \leq & \frac{m-N}{20} \int_{\mathbb{R}^{N}}\left|\frac{W(\tau)}{\tilde{W}(\tau)}\right|^{2} \tilde{W}(\tau) \\
& +\frac{1}{3} \int_{\mathbb{R}^{N}}\left|\nabla\left(\frac{W(\tau)}{\tilde{W}(\tau)}\right)\right|^{2} \tilde{W}(\tau)+C \ell\left(U_{\mathrm{ini}}\right)^{2} .
\end{aligned}
$$

Similarly, the calculations of the fourth step in the proof of Proposition 3.2 yield

$$
\begin{aligned}
e^{(1-N) \tau} \int_{\mathbb{R}^{N}} & |W(\tau, x)|^{2}\left|\nabla_{x}\left(\frac{W(\tau, x)}{\tilde{W}(\tau, x)}\right)\right| d x \\
\leq & C\|W(\tau)\|_{L^{1}\left(\mathbb{R}^{N}\right)}^{1 / N}\left[\int_{\mathbb{R}^{N}}\left|\frac{W(\tau)}{\tilde{W}(\tau)}\right|^{2} \tilde{W}(\tau)+\int_{\mathbb{R}^{N}}\left|\nabla\left(\frac{W(\tau)}{\tilde{W}(\tau)}\right)\right|^{2} \tilde{W}(\tau)\right] \\
\leq & C \ell\left(U_{\mathrm{ini}}\right)^{1 / N}\left[\int_{\mathbb{R}^{N}}\left|\frac{W(\tau)}{\tilde{W}(\tau)}\right|^{2} \tilde{W}(\tau)+\int_{\mathbb{R}^{N}}\left|\nabla\left(\frac{W(\tau)}{\tilde{W}(\tau)}\right)\right|^{2} \tilde{W}(\tau)\right] .
\end{aligned}
$$

The additional terms coming from the estimation of $b$ can be easily bounded thanks to the Cauchy-Schwarz inequality. For $\tau \geq \tau_{0}$, and $\tau_{0}$ large enough, we have

$$
\begin{array}{r}
C e^{-N \tau} \int_{\mathbb{R}^{N}}|W(\tau)|\left|\nabla_{y}\left(\frac{W(\tau)}{\tilde{W}(\tau)}\right)\right|+C e^{(1-2 N) \tau} \int_{\mathbb{R}^{N}}\left|U^{\mathrm{app}}(\tau)\right|^{3}\left|\nabla\left(\frac{W(\tau)}{\tilde{W}(\tau)}\right)\right| \\
\leq \frac{m-N}{20} \int_{\mathbb{R}^{N}}\left|\frac{W(\tau)}{\tilde{W}(\tau)}\right|^{2} \tilde{W}(\tau)+\frac{1}{3} \int_{\mathbb{R}^{N}}\left|\nabla\left(\frac{W(\tau)}{\tilde{W}(\tau)}\right)\right|^{2} \tilde{W}(\tau)+C .
\end{array}
$$

Eventually, using the Cauchy-Schwarz inequality together with the bound (4.6), we infer that

$$
\begin{aligned}
\int_{\mathbb{R}^{N}}\left|\frac{W(\tau, x)}{\tilde{W}(\tau, x)}\right|\left|U^{\mathrm{rem}}(\tau, x)\right| d x & \leq\left\|U^{\mathrm{rem}}(\tau)\right\|_{L^{2}\left(\tilde{W}(\tau)^{-1}\right)}\left(\int_{\mathbb{R}^{N}}\left|\frac{W(\tau)}{\tilde{W}(\tau)}\right|^{2} \tilde{W}(\tau)\right)^{1 / 2} \\
& \leq C\left\|U^{\mathrm{rem}}(\tau)\right\|_{L^{2}\left(e^{\left.\gamma|x|^{2}\right)}\right.}\left(\int_{\mathbb{R}^{N}}\left|\frac{W(\tau)}{\tilde{W}(\tau)}\right|^{2} \tilde{W}(\tau)\right)^{1 / 2} \\
& \leq C e^{-\tau}\left(\int_{\mathbb{R}^{N}}\left|\frac{W(\tau)}{\tilde{W}(\tau)}\right|^{2} \tilde{W}(\tau)\right)^{1 / 2} \\
& \leq C+\frac{m-N}{20} \int_{\mathbb{R}^{N}}\left|\frac{W(\tau)}{\tilde{W}(\tau)}\right|^{2} \tilde{W}(\tau) .
\end{aligned}
$$

Gathering all the terms, we deduce that there exists a constant $C_{m}$, depending only on $N$ and $m$, such that if $\ell\left(U_{\text {ini }}\right) \leq C_{m}$, then for all $\tau \geq \tau_{0}$,

$$
\frac{d}{d \tau} \int_{\mathbb{R}^{N}}\left|\frac{W(\tau)}{\tilde{W}(\tau)}\right|^{2} \tilde{W}(\tau)+\frac{m-N}{20} \int_{\mathbb{R}^{N}}\left|\frac{W(\tau)}{\tilde{W}(\tau)}\right|^{2} \tilde{W}(\tau)+\int_{\mathbb{R}^{N}}\left|\nabla\left(\frac{W(\tau)}{\tilde{W}(\tau)}\right)\right|^{2} \tilde{W}(\tau) \leq C .
$$

Compactness of a subsequence $W\left(\tau_{n}\right)$ follows. Hence the $\omega$-limit set is nonempty, and thus $\ell\left(U_{\text {ini }}\right)=0$. 


\section{Appendix}

Lemma A.1. Assume that the flux A satisfies (1.4), (1.5). Let $v \in W^{1, \infty}\left(\mathbb{T}^{N}\right)$ be a periodic stationary solution of $(1.1)$, and let $u \in L_{\mathrm{loc}}^{\infty}\left([0, \infty), L^{\infty}\left(\mathbb{R}^{N}\right)\right) \cap \mathcal{C}\left([0, \infty), L_{\mathrm{loc}}^{1}\left(\mathbb{R}^{N}\right)\right)$ be the unique solution of (1.1) with initial data $u_{\mathrm{ini}} \in v(y)+L^{1} \cap L^{\infty}\left(\mathbb{R}^{N}\right)$. Then $u \in L^{\infty}\left([0, \infty) \times \mathbb{R}^{N}\right)$.

Proof. This result was proved in [8] in the case $N=1$. When $N \geq 2$, the proof goes along the same lines; the only difference lies in the use of the Sobolev embeddings, which depend on the dimension. Hence we merely recall here the main steps of the proof, with an emphasis on the case $N \geq 2$.

In the rest of the proof, we set $f(t, y)=u(t, y)-v(y)$. Then $f$ solves the equation

$$
\partial_{t} f+\operatorname{div}_{y} B(y, f)-\Delta_{y} f=0,
$$

and according to (1.5) the flux $B$ is such that for all $f \in \mathbb{R}$,

$$
\left|\operatorname{div}_{y} B(y, f)\right| \leq C\left(|f|+|f|^{n}\right), \quad\left|\partial_{f} B(y, f)\right| \leq C\left(|f|+|f|^{n}\right),
$$

where the exponent $n$ is such that $n<(N+2) / N$. Moreover,

$$
\|f(t)\|_{L^{1}\left(\mathbb{R}^{N}\right)} \leq\left\|u_{\mathrm{ini}}-v\right\|_{L^{1}\left(\mathbb{R}^{N}\right)} \quad \forall t \geq 0 .
$$

For $q \geq 1$ arbitrary, multiply (4.7) by $f|f|^{q-1}$, and integrate over $\mathbb{R}^{N}$. Using a few integrations by parts (see [8]), we are led to

$$
\frac{d}{d t} \int_{\mathbb{R}^{N}}|f|^{q+1}+\left.\left.c_{q} \int_{\mathbb{R}^{N}}\left|\nabla_{y}\right| f\right|^{(q+1) / 2}\right|^{2} \leq C_{q}\left(\int_{\mathbb{R}^{N}}|f|^{q+1}+\int_{\mathbb{R}^{N}}|f|^{q+n}\right) .
$$

We then use Sobolev embeddings in order to control the $L^{q+1}$ and $L^{q+n}$ norms on the right-hand side. We distinguish between the cases $N=2$ and $N \geq 3$, since the space $H^{1}$ is critical in dimension two.

- If $N=2$, then $H^{1}\left(\mathbb{R}^{2}\right) \subset L^{p}\left(\mathbb{R}^{2}\right)$ for all $p \in[2, \infty)$. Interpolating $L^{q+n}$ between $L^{1}$ and $L^{p}$ for some $p$ sufficiently large, we have, using the Gagliardo-Nirenberg-Sobolev inequality,

$$
\begin{aligned}
\|f\|_{L^{q+n}\left(\mathbb{R}^{2}\right)} & \leq\|f\|_{L^{1}\left(\mathbb{R}^{2}\right)}^{\theta}\|f\|_{L^{p}\left(\mathbb{R}^{2}\right)}^{1-\theta} \quad \text { with } \frac{1}{q+n}=\frac{\theta}{1}+\frac{1-\theta}{p} \\
& \leq\|f\|_{L^{1}\left(\mathbb{R}^{2}\right)}^{\theta}\left\||f|^{(q+1) / 2}\right\|_{L^{2 p /(q+1)}\left(\mathbb{R}^{2}\right)}^{2(1-\theta) /(q+1)} \\
& \leq C_{p}\|f\|_{L^{1}\left(\mathbb{R}^{2}\right)}^{\theta}\|f\|_{L^{(q+1) / 2}\left(\mathbb{R}^{2}\right)}^{(1-\theta)(q+1) / 2 p}\left\|\nabla|f|^{(q+1) / 2}\right\|_{L^{2}\left(\mathbb{R}^{2}\right)}^{(1-\theta)\left(\frac{2}{q+1}-\frac{1}{p}\right)}
\end{aligned}
$$

Notice that

$$
2 \frac{q+n}{q+1}(1-\theta)=2 \frac{q+n-1}{(q+1)(1-1 / p)}
$$


and

$$
\frac{q+n-1}{q+1}<1 \quad \forall q \geq 1
$$

since $n<(N+2) / N$. Thus, we choose $p>1$ such that

$$
0<(q+n)(1-\theta)\left(\frac{2}{q+1}-\frac{1}{p}\right)<2 .
$$

Young's inequality then implies that for all $\lambda>0$, there exists a constant $C_{\lambda, q}$ and exponents $q_{1}, q_{2}$ such that

$$
\int_{\mathbb{R}^{N}}|f|^{q+n} \leq \lambda\left\|\nabla|f|^{(q+1) / 2}\right\|_{L^{2}\left(\mathbb{R}^{2}\right)}^{2}+C_{\lambda, q}\left(\|f\|_{L^{1}\left(\mathbb{R}^{2}\right)}^{q_{1}}+\|f\|_{L^{(q+1) / 2}\left(\mathbb{R}^{2}\right)}^{q_{2}}\right) .
$$

The other term on the right-hand side of (4.8) can be bounded in a similar fashion: we have, for all $\lambda>0$,

$$
\int_{\mathbb{R}^{N}}|f|^{q+1} \leq \lambda\left\|\nabla|f|^{(q+1) / 2}\right\|_{L^{2}\left(\mathbb{R}^{2}\right)}^{2}+C_{\lambda, q}\left(\|f\|_{L^{1}\left(\mathbb{R}^{2}\right)}^{q_{3}}+\|f\|_{L^{(q+1) / 2}\left(\mathbb{R}^{2}\right)}^{q_{4}}\right),
$$

for some exponents $q_{3}, q_{4}$ which can be explicitly computed. Choosing an appropriate parameter $\lambda$, we infer that there exist $q_{1}, q_{2}, q_{3}, q_{4}>0$ such that

$$
\begin{aligned}
\frac{d}{d t} \int_{\mathbb{R}^{N}}|f|^{q+1} & +\left.\left.c_{q} \int_{\mathbb{R}^{N}}\left|\nabla_{y}\right| f\right|^{(q+1) / 2}\right|^{2} \\
\leq & C_{q}\left(\|f\|_{L^{1}\left(\mathbb{R}^{2}\right)}^{q_{1}}+\|f\|_{L^{(q+1) / 2}\left(\mathbb{R}^{2}\right)}^{q_{2}}+\|f\|_{L^{1}\left(\mathbb{R}^{2}\right)}^{q_{3}}+\|f\|_{L^{(q+1) / 2}\left(\mathbb{R}^{2}\right)}^{q_{4}}\right) .
\end{aligned}
$$

Using (4.10) one more time leads to

$$
\begin{aligned}
\frac{d}{d t} \int_{\mathbb{R}^{N}}|f|^{q+1} & +c_{q} \int_{\mathbb{R}^{N}}|f|^{q+1} \\
& \leq C_{q}\left(\|f\|_{L^{1}\left(\mathbb{R}^{2}\right)}^{q_{1}}+\|f\|_{L^{(q+1) / 2}\left(\mathbb{R}^{2}\right)}^{q_{2}}+\|f\|_{L^{1}\left(\mathbb{R}^{2}\right)}^{q_{3}}+\|f\|_{L^{(q+1) / 2}\left(\mathbb{R}^{2}\right)}^{q_{4}}\right) .
\end{aligned}
$$

Using a Gronwall-type argument, we infer that for all $q \geq 1$,

$$
f \in L^{\infty}\left([0, \infty), L^{(q+1) / 2}\left(\mathbb{R}^{2}\right)\right) \Rightarrow f \in L^{\infty}\left([0, \infty), L^{q+1}\left(\mathbb{R}^{2}\right)\right) .
$$

Since $f \in L^{\infty}\left([0, \infty), L^{1}\left(\mathbb{R}^{2}\right)\right)$, we infer by induction on $q$ that $f \in L^{\infty}\left([0, \infty), L^{q}\left(\mathbb{R}^{2}\right)\right)$ for all $q \in[1, \infty)$.

- When $N \geq 3$, we use the Sobolev embedding $H^{1}\left(\mathbb{R}^{N}\right) \subset L^{p^{*}}\left(\mathbb{R}^{N}\right)$, where

$$
p^{*}=\frac{2 N}{N-2} \text {. }
$$

Interpolating $L^{q+n}$ between $L^{1}$ and $L^{p^{*}(q+1) / 2}$, we obtain

$$
\begin{aligned}
& \|f\|_{L^{q+n}\left(\mathbb{R}^{N}\right)} \leq\|f\|_{L^{p^{*}(q+1) / 2}\left(\mathbb{R}^{N}\right)}^{\theta}\|f\|_{L^{1}\left(\mathbb{R}^{N}\right)}^{1-\theta} \leq\left\||f|^{(q+1) / 2}\right\|_{L^{p^{*}\left(\mathbb{R}^{N}\right)}}^{2 \theta /(q+1)}\|f\|_{L^{1}\left(\mathbb{R}^{N}\right)}^{1-\theta} \\
& \leq C\left\|\nabla|f|^{(q+1) / 2}\right\|_{L^{2}\left(\mathbb{R}^{N}\right)}^{2 \theta /(q+1)}\|f\|_{L^{1}\left(\mathbb{R}^{N}\right)}^{1-\theta},
\end{aligned}
$$


where the parameter $\theta \in(0,1)$ is given by

$$
\frac{1}{q+n}=\frac{2 \theta}{p^{*}(q+1)}+\frac{1-\theta}{1} .
$$

It can be checked that

$$
n<\frac{N+2}{N} \Rightarrow \frac{\theta(q+n)}{q+1}<1 .
$$

Hence (4.9) holds when $N \geq 3$. Inequality (4.10) is proved with similar arguments. As in the two-dimensional case, we deduce that $f \in L^{\infty}\left([0, \infty), L^{q}\left(\mathbb{R}^{N}\right)\right)$ for all $q \in[1, \infty)$.

Using Theorem 8.1 in Chapter III of [16] (see [8] for details), we infer eventually that $f \in L^{\infty}\left([0, \infty) \times \mathbb{R}^{N}\right)$.

Lemma A.2. Let $M>M^{\prime}$ be arbitrary. Then

$$
F_{M}(y)>F_{M^{\prime}}(y) \quad \forall y \in \mathbb{R}^{N} .
$$

As a consequence,

$$
\left\|F_{M}-F_{M^{\prime}}\right\|_{L^{1}\left(\mathbb{R}^{N}\right)}=M-M^{\prime} .
$$

Proof. The arguments are exactly the ones which lead to the uniqueness of stationary solutions of (2.13), (2.15), and they can be found in [1]. We recall the main steps below for the reader's convenience.

Let $F:=F_{M}-F_{M^{\prime}}$. Then $F \in L^{1} \cap \mathcal{C}^{2}\left(\mathbb{R}^{N}\right)$, and $\int_{\mathbb{R}^{N}} F>0$. Hence the set

$$
\Theta:=\left\{x \in \mathbb{R}^{N}: F(x)>0\right\}
$$

is nonempty. The idea is to prove that $F_{+}=F \mathbf{1}_{\Theta}$ satisfies a linear elliptic equation; since $F_{+} \geq 0$, the strong maximum principle entails that $F_{+}$cannot vanish anywhere, and thus $F_{+}(x)>0$ for all $x \in \mathbb{R}^{N}$.

Let us now derive an equation on $F_{+}$. Subtracting the equations on $F_{M}$ and $F_{M^{\prime}}$, we have

$$
-\sum_{1 \leq i, j \leq N} \eta_{i, j} \frac{\partial^{2} F}{\partial x_{i} \partial x_{j}}+\operatorname{div}_{x}(b F)=0,
$$

where

$$
b(x)=a\left(F_{M}(x)+F_{M^{\prime}}(x)\right)-x, \quad x \in \mathbb{R}^{N} ;
$$

remember that $a=0$ if $N \geq 2$. Since $F \in H^{2}\left(\mathbb{R}^{N}\right)$, we have

$$
\operatorname{div}_{x}(b F) \mathbf{1}_{\Theta}=\operatorname{div}_{x}\left(b F_{+}\right)
$$

almost everywhere. Thus, we obtain

$$
-\sum_{1 \leq i, j \leq N} \eta_{i, j} \mathbf{1}_{\Theta} \frac{\partial^{2} F}{\partial x_{i} \partial x_{j}}+\operatorname{div}_{x}\left(b F_{+}\right)=0 .
$$


Integrating the above equation on $\mathbb{R}^{N}$ leads to

$$
\int_{\Theta} \sum_{1 \leq i, j \leq N} \eta_{i, j} \frac{\partial^{2} F}{\partial x_{i} \partial x_{j}}=0
$$

Let us now perform the change of variables (2.18), which changes the matrix $\eta$ into identity: setting $\tilde{F}(y)=F(P y)$, and $\tilde{\Theta}:=\{\tilde{F}>0\}$, we infer

$$
\int_{\tilde{\Theta}} \Delta_{y} \tilde{F}=C \int_{\Theta} \sum_{1 \leq i, j \leq N} \eta_{i, j} \frac{\partial^{2} F}{\partial x_{i} \partial x_{j}}=0 .
$$

Moreover, $\tilde{F} \in H^{2} \cap W^{2,1}\left(\mathbb{R}^{N}\right)$, and thus Lemma 7 in [1] applies. We deduce that

$$
\Delta_{y}\left(\tilde{F} \mathbf{1}_{\tilde{\Theta}}\right)=\mathbf{1}_{\tilde{\Theta}} \Delta_{y} \tilde{F},
$$

and thus

$$
\sum_{1 \leq i, j \leq N} \eta_{i, j} \mathbf{1}_{\Theta} \frac{\partial^{2} F}{\partial x_{i} \partial x_{j}}=\sum_{1 \leq i, j \leq N} \eta_{i, j} \frac{\partial^{2} F_{+}}{\partial x_{i} \partial x_{j}} .
$$

Eventually, $F_{+}$solves the elliptic equation

$$
-\sum_{1 \leq i, j \leq N} \eta_{i, j} \frac{\partial^{2} F_{+}}{\partial x_{i} \partial x_{j}}+\operatorname{div}_{x}\left(b F_{+}\right)=0,
$$

with $b \in L_{\mathrm{loc}}^{\infty}\left(\mathbb{R}^{N}\right)$. Using either a unique continuation principle or Harnack's inequality (see [13, Theorem 8.20]), we infer that if $F_{+}$vanishes at some point $x$ in $\mathbb{R}^{N}$, then $F_{+}$ is identically zero on $\mathbb{R}^{N}$, which is absurd. Hence $F_{+}(x)>0$ for all $x \in \mathbb{R}^{N}$, and thus $\mathbb{R}^{N} \backslash \Theta=\emptyset$, which means that $F(x)>0$ for all $x \in \mathbb{R}^{N}$.

Acknowledgments. I wish to thank Adrien Blanchet, Jean Dolbeault, and Michał Kowalczyk, for very fruitful and stimulating discussions.

\section{References}

[1] Aguirre, J., Escobedo, M., Zuazua, E.: Self-similar solutions of a convection diffusion equation and related semilinear elliptic problems. Comm. Partial Differential Equations 15, 139157 (1990) Zbl 0708.35030 MR 1032626

[2] Allaire, G.: Homogenization and two-scale convergence. SIAM J. Math. Anal. 23, 1482-1518 (1992) Zbl 0770.35005 MR 1185639

[3] Bensoussan, A., Lions, J.-L., Papanicolaou, G.: Asymptotic Analysis for Periodic Structures. Stud. Math. Appl. 5, North-Holland, Amsterdam (1978) Zbl 0404.35001 MR 0503330

[4] Blanchet, A., Dolbeault, J., Kowalczyk, M.: Travelling fronts in stochastic Stokes' drifts. Phys. A 387, 5741-5751 (2008)

[5] Blanchet, A., Dolbeault, J., Kowalczyk, M.: Stochastic Stokes' drift, homogenized functional inequalities, and large time behavior of brownian ratchets. SIAM J. Math. Anal. 41, 46-76 (2009) Zbl 1196.26023 MR 2505853 
[6] Brezis, H.: Analyse fonctionnelle. Masson, Paris (1983) Zbl 0511.46001 MR 0697382

[7] Dalibard, A.-L.: Homogenization of a quasilinear parabolic equation with vanishing viscosity. J. Math. Pures Appl. (9) 86, 133-154 (2006) Zbl 1121.35014 MR 2247455

[8] Dalibard, A.-L.: Long time behavior of parabolic scalar conservation laws with space periodic flux. Indiana Univ. Math. J. 59, 257-300 (2010) Zbl 1204.35045 MR 2666480

[9] Dautray, R., Lions, J.-L.: Analyse mathématique et calcul numérique pour les sciences et les techniques. Vol. 5. Masson, Paris, (1988) Zbl 0664.47003 MR 0944303

[10] Di Francesco, M., Markowich, P. A.: Entropy dissipation and Wasserstein metric methods for the viscous Burgers' equation: convergence to diffusive waves. In: Partial Differential Equations and Inverse Problems, Contemp. Math. 362, Amer. Math. Soc., Providence, RI, 145-165 (2004) Zbl 1062.35111 MR 2091496

[11] Escobedo, M., Vázquez, J. L., Zuazua, E.: Asymptotic behaviour and source-type solutions for a diffusion-convection equation. Arch. Ration. Mech. Anal. 124, 43-65 (1993) Zbl 0807.35059 MR 1233647

[12] Escobedo, M., Zuazua, E.: Large time behavior for convection-diffusion equations in $\mathbf{R}^{N}$. J. Funct. Anal. 100, 119-161 (1991) Zbl 0762.35011 MR 1124296

[13] Gilbarg, D., Trudinger, N. S.: Elliptic Partial Differential Equations of Second Order. Classics in Math., Springer, Berlin (2001) Zbl 1042.35002 MR 1814364

[14] Goudon, T., Poupaud, F.: Homogenization of transport equations: weak mean field approximation. SIAM J. Math. Anal. 36, 856-881 (2004/05) Zbl 1077.35019 MR 2111918

[15] Kruzhkov, S. N.: First order quasilinear equations in several independent variables. Math. USSR Sb. 10, 217-243 (1970) Zbl 0215.16203

[16] Ladyženskaja, O. A., Solonnikov, V. A., Ural'ceva, N. N.: Linear and Quasilinear Equations of Parabolic Type. Transl. Math. Monogr. 23, Amer. Math. Soc., Providence, RI (1967) Zbl 0164.12302 MR 0241822

[17] LaSalle, J. P.: The Stability of Dynamical Systems. SIAM, Philadelphia, PA (1976) Zbl 0364.93002 MR 0481301

[18] Le Blanc, V.: $L^{1}$-stability of periodic stationary solutions of scalar convection-diffusion equations. J. Differential Equations 247, 1746-1761 (2009) Zbl 1180.35093 MR 2553857

[19] Michel, P., Mischler, S., Perthame, B.: General relative entropy inequality: an illustration on growth models. J. Math. Pures Appl. (9) 84, 1235-1260 (2005) Zbl 1085.35042 MR 2162224

[20] Osher, S., Ralston, J.: $L^{1}$ stability of travelling waves with applications to convective porous media flow. Comm. Pure Appl. Math. 35, 737-749 (1982) Zbl 0479.35053 MR 0673828

[21] Serre, D.: Systems of Conservation Laws. 1. Cambridge Univ. Press, Cambridge (1999) Zbl 0930.35002 MR 1707279

[22] Serre, D.: $L^{1}$-stability of nonlinear waves in scalar conservation laws. In: Evolutionary Equations. Vol. I, Handbook Diff. Equations, North-Holland, Amsterdam, 473-553 (2004) Zbl 1079.35064 MR 2103702

[23] Zuazua, E.: Weakly nonlinear large time behavior in scalar convection-diffusion equations. Differential Integral Equations 6, 1481-1491 (1993) Zbl 0805.35054 MR 1235206

[24] Zuazua, E.: A dynamical system approach to the self-similar large time behavior in scalar convection-diffusion equations. J. Differential Equations 108, 1-35 (1994) Zbl 0802.35074 MR 1268349 